\title{
Scalar Multivariate Subdivision Schemes and Box Splines
}

\author{
Maria Charina $^{\mathrm{a}, *}$, Costanza Conti ${ }^{\mathrm{b}}$, Kurt Jetter $^{\mathrm{c}}$, Georg Zimmermann ${ }^{\mathrm{c}}$ \\ ${ }^{a}$ Fakultät für Mathematik, TU Dortmund, D-44221 Dortmund, Germany \\ ${ }^{b}$ Dipartimento di Energetica, Università di Firenze, Via C. Lombroso 6/17, I-50134 \\ Firenze, Italy \\ ${ }^{c}$ Institut für Angewandte Mathematik und Statistik, Universität Hohenheim, D-70593 \\ Stuttgart, Germany
}

\begin{abstract}
We study scalar $d$-variate subdivision schemes, with dilation matrix $2 I$, satisfying the sum rules of order $k$. Using the results of Möller and Sauer, stated for general expanding dilation matrices, we characterize the structure of the mask symbols of such schemes by showing that they must be linear combinations of shifted box spline generators of some quotient polynomial ideal. The directions of the corresponding box splines are columns of certain unimodular matrices. The quotient ideal is determined by the given order of the sum rules or, equivalently, by the order of the zero conditions.

The results presented in this paper open a way to a systematic study of subdivision schemes, since box spline subdivisions turn out to be the building blocks of any reasonable multivariate subdivision scheme.

As in the univariate case, the characterization we give is the proper way of matching the smoothness of the box spline building blocks with the order of polynomial reproduction of the corresponding subdivision scheme. However, due to the interaction of the building blocks, convergence and smoothness properties may change, if several convergent schemes are combined.
\end{abstract}

The results are illustrated with several examples.

Keywords: Subdivision schemes, box splines, ideals

\section{Introduction}

Subdivision schemes are efficient iterative procedures for generating finer and finer grids of points in $\mathbb{R}^{d}$ and are used to design smooth curves or surfaces. Starting with some initial grid of points, a binary scalar subdivision scheme

\footnotetext{
${ }^{*}$ Corresponding author

Email addresses: maria.charina@uni-dortmund.de (Maria Charina), costanza.conti@unifi.it (Costanza Conti), Kurt.Jetter@uni-hohenheim.de (Kurt Jetter), Georg.Zimmermann@uni-hohenheim.de (Georg Zimmermann)
} 
computes the coordinates of the finer grid points $\mathrm{d}^{(r+1)}$, inheriting the topology of the coarser ones, via local averages

$$
\mathrm{d}^{(r+1)}=\mathcal{S}_{\mathrm{a}} \mathrm{d}^{(r)}=\sum_{\boldsymbol{\beta} \in \mathbb{Z}^{d}} \mathrm{~d}_{\boldsymbol{\beta}}^{(r)} \mathrm{a}_{\boldsymbol{\alpha}-2 \boldsymbol{\beta}}, \quad r \geq 0 .
$$

These averaging rules depend on the coefficients of the corresponding subdivision mask a $=\left(\mathrm{a}_{\boldsymbol{\alpha}}\right)_{\boldsymbol{\alpha} \in \mathbb{Z}^{d}}$ which we assume to be some finitely supported sequence of real numbers. If the mask is chosen appropriately, the grids with the vertices $\mathrm{d}^{(r)}$, as $r$ goes to infinity, can be interpreted as approximations of the values of a limiting curve or surface. The locality of the method and its algorithmic simplicity ensure that the subdivision recursion is fast, efficient, and easy to implement. These features and the connection between subdivision and other multiresolution methods have led to an increasing popularity of subdivision in computer graphics, in computer aided geometrical design, and in wavelet and frame constructions. For more details on subdivision we refer the interested reader to the pioneering work in [4, 17] or to the more recent survey [19] and the references therein. For basic details on wavelet and frame constructions see, e.g., 7, 13, 32].

In general, the limit of a convergent subdivision scheme is not known analytically. Nevertheless, various analytical properties of the limit can be read off the mask symbol

$$
\mathrm{a}(\boldsymbol{z})=\sum_{\boldsymbol{\alpha} \in \mathbb{Z}^{d}} \mathrm{a}_{\boldsymbol{\alpha}} \boldsymbol{z}^{\boldsymbol{\alpha}}, \quad \boldsymbol{z} \in(\mathbb{C} \backslash\{0\})^{d},
$$

which - due to the finite support of the mask - is a $d$-variate Laurent polynomial. In the univariate setting, the properties of convergent subdivision schemes are well-understood. The symbol a $(z)$ of any convergent univariate subdivision scheme possesses the Factorization Property: it can be written as

$$
\mathrm{a}(z)=2 \cdot \sigma(z) \cdot\left(\frac{1+z}{2}\right)^{k}, \quad \text { with } \quad \sigma(1)=1,
$$

for some integer $k \geq 1$, with the Laurent polynomial $\sigma(z)$ satisfying some additional properties to ensure the convergence and smoothness of the subdivision limit, see 14, 29]. Since the coefficients of $\sigma$ sum to 1, this Factorization Property tells us that convergence of the subdivision scheme implies that its mask can be written as an affine combination of shifted versions of B-spline symbols $B_{k}(z)=(1+z)^{k} / 2^{k-1}$. Thus, any reasonable univariate subdivision scheme uses B-spline symbols as its building blocks, a fact which is very crucial for designing new efficient subdivision processes, see e.g., [11, 15, 16, 20].

In this paper we are going to present results which aim at replacing the Factorization Property of univariate schemes by a Decomposition Property of multivariate schemes, see Theorems $\mathrm{A}-\mathrm{C}$ below. Theorem $\mathrm{C}$ deals with the case $d=2$ and is crucial for studying the properties of subdivision schemes. It tells 
us that the mask symbol of any reasonable bivariate subdivision scheme can be decomposed as

$$
\mathrm{a}\left(z_{1}, z_{2}\right)=4 \cdot \sum_{B_{\alpha, \beta, \gamma}^{\#} \in \mathrm{I}_{k}} \lambda_{\alpha, \beta, \gamma} \cdot \sigma_{\alpha, \beta, \gamma}\left(z_{1}, z_{2}\right) \cdot B_{\alpha, \beta, \gamma}^{\#}\left(z_{1}, z_{2}\right),
$$

where $\sum \lambda_{\alpha, \beta, \gamma}=1$, and the symbols $\sigma_{\alpha, \beta, \gamma}\left(z_{1}, z_{2}\right)$ are Laurent polynomials normalized by $\sigma_{\alpha, \beta, \gamma}(1,1)=1$. Here,

$$
B_{\alpha, \beta, \gamma}^{\#}\left(z_{1}, z_{2}\right)=\left(\frac{1+z_{1}}{2}\right)^{\alpha}\left(\frac{1+z_{2}}{2}\right)^{\beta}\left(\frac{1+z_{1} z_{2}}{2}\right)^{\gamma}, \quad \alpha, \beta, \gamma \in \mathbb{N}_{0},
$$

are the normalized mask symbols of three-directional box splines, $k$ refers to the order of polynomial reproduction of the subdivision operator $S_{\mathrm{a}}$-in the wavelet literature also denoted as order of accuracy of the mask - and the list $\mathrm{I}_{k}$ is as in Theorem 3.2. The advantage of such a decomposition result is obvious, not only for the purpose of classifying the zoo of subdivision schemes, but also as a starting point for constructing new schemes or for enhancing the properties of the schemes according to specific requirements.

We believe that the use of the building blocks $B_{\alpha, \beta, \gamma}^{\#}$ in the above decomposition is the appropriate generalization to the bivariate case of the normalized univariate B-spline symbols, since $B_{\alpha, \beta, \gamma}^{\#}$ correspond to a class of well-known bivariate spline functions whose order of global smoothness and whose order of polynomial reproduction match in the same way as in the univariate case, see the notes at the end of Section 3.1. We consider, therefore, this paper to be also an interesting contribution to the box spline literature.

The methods employed in this paper are of algebraic nature, as well as other results on subdivision dealing with polynomial reproduction, see [4, Chapter 6]. It is well-known that the necessary conditions for the convergence of a subdivision scheme are equivalent to the fact that the mask symbol a $(\boldsymbol{z})$ is a properly normalized element of a certain ring of Laurent polynomials: the symbol must belong to the ideal $\mathcal{I}$, or to its power $\mathcal{I}^{k}$, depending on the order $k$ of the polynomial reproduction of the scheme. For definitions of $\mathcal{I}^{k}, k \in \mathbb{N}$, see (2.1) and (1.6), or see the zero conditions of order $k$ in (1.7). This algebraic property of $\mathrm{a}(\boldsymbol{z})$ has been studied in detail in the papers by Sauer 30, 31 and by Möller and Sauer [28], which also motivated us to consider these ideals once again. Note that the Factorization Property says that a $(\boldsymbol{z})$ belongs to the principal ideal generated by some B-spline symbol, while the Decomposition Property tells us that $\mathrm{a}(\boldsymbol{z})$ is in the ideal generated by a particular set of box spline symbols. Möller and Sauer have given other sets of generators for these ideals, putting the emphasis on the algebraic properties of these generators. We consider it one of our main achievements that we were able to relate the generators of the ideals $\mathcal{I}^{k}$ to a well-known class of spline functions, see 2]. Box spline subdivision has been studied thoroughly in the literature, see [3, 8], where such box spline schemes also appear under a different name. In the three-directional case, for example, their mask symbols are given by $4 \cdot B_{\alpha, \beta, \gamma}^{\#}\left(z_{1}, z_{2}\right)$. 
Starting with Section 2, we restrict ourselves to working with the ideals of the ring of $d$-variate polynomials instead of the ring of Laurent polynomials. Note that any Laurent polynomial can be shifted to produce a polynomial. It is done by multiplying the Laurent polynomial with a factor $z^{\alpha}$, i.e., a unit in the ring of Laurent polynomials. This results in a shift of the support of the mask. Such a shift does neither affect the convergence and regularity of the scheme, nor does it change the zero conditions of any order. We, therefore, assume that the mask is supported in $\mathbb{N}_{0}^{d}$.

The paper is organized as follows: in Section 1 we introduce some notation and background on scalar subdivision schemes with dilation matrix $2 I$. In Section 2 we show how the zero conditions of order $k=1$ on the mask symbol $\mathrm{a}(\boldsymbol{z})$ determine the structure of the symbol of any convergent binary subdivision scheme. This result is given in Theorem A and is a crucial step toward understanding the case $k>1$. As in the multivariate case the Factorization Property is replaced by the Decomposition Property, it is important to reduce the number of the terms of this decomposition or, equivalently, to minimize the number of the generators for $\mathcal{I}$, see Theorem 2.6. Theorem [2.11. Section 3 contains our main results, Theorems $\mathrm{B}$ and $\mathrm{C}$, dealing with powers of $\mathcal{I}$. The fact that the ideal $\mathcal{I}^{k}$ is generated by the appropriate products of the elements of $\mathcal{I}$ makes the proof of Theorem B straightforward, if one is not interested in reducing the number of terms of the corresponding decomposition. We address the latter issue only in the case $d=2$, as this case is of special interest in subdivision. The corresponding result is stated in Theorem C. In Section 4 we illustrate the result of Theorem $\mathrm{C}$ with several bivariate examples.

This paper is an extended version of the technical report [5] which can be obtained from the first author.

\section{Background on subdivision and notation}

A scalar $d$-variate subdivision scheme is given by a scalar $\mathbb{Z}^{d}$-indexed sequence $\mathrm{a}=\left(\mathrm{a}_{\boldsymbol{\alpha}}\right)_{\boldsymbol{\alpha} \in \mathbb{Z}^{d}}$, the so-called mask, defining the subdivision operator $\mathcal{S}_{\mathrm{a}}$ on data sequences $\mathrm{d}=\left(\mathrm{d}_{\boldsymbol{\alpha}}\right)_{\boldsymbol{\alpha} \in \mathbb{Z}^{d}} \in \ell\left(\mathbb{Z}^{d}\right)$ as follows:

$$
\left(\mathcal{S}_{\mathrm{a}} \mathrm{d}\right)_{\boldsymbol{\alpha}}=\sum_{\boldsymbol{\beta} \in \mathbb{Z}^{d}} \mathrm{~d}_{\boldsymbol{\beta}} \mathrm{a}_{\boldsymbol{\alpha}-2 \boldsymbol{\beta}}, \quad \boldsymbol{\alpha} \in \mathbb{Z}^{d} .
$$

We assume that the mask is finite, i.e., only finitely many coefficients $\mathrm{a}_{\boldsymbol{\alpha}}$ are non-zero.

In our study we use the following symbol notation. For a finitely supported sequence $\mathrm{c}=\left(\mathrm{c}_{\boldsymbol{\alpha}}\right)_{\boldsymbol{\alpha} \in \mathbb{Z}^{d}}$, its symbol is given by the Laurent polynomial

$$
\mathrm{c}(\boldsymbol{z})=\sum_{\boldsymbol{\alpha} \in \mathbb{Z}^{d}} \mathrm{c}_{\boldsymbol{\alpha}} \boldsymbol{z}^{\boldsymbol{\alpha}}
$$

with $\boldsymbol{z}=\left(z_{1}, \ldots, z_{d}\right) \in(\mathbb{C} \backslash\{0\})^{d}$ and, in the multi-index notation,

$$
\boldsymbol{z}^{\boldsymbol{\alpha}}=z_{1}^{\alpha_{1}} z_{2}^{\alpha_{2}} \cdot \ldots \cdot z_{d}^{\alpha_{d}}, \quad \text { for } \boldsymbol{\alpha}=\left(\alpha_{1}, \ldots, \alpha_{d}\right) \in \mathbb{Z}^{d} .
$$


In the symbol notation, the subdivision step in (1.1) is described by the identity

$$
\left(\mathcal{S}_{\mathrm{a}} \mathrm{d}\right)(\boldsymbol{z})=\mathrm{d}\left(\boldsymbol{z}^{2}\right) \mathrm{a}(\boldsymbol{z}), \quad \boldsymbol{z}^{2}=\left(z_{1}^{2}, z_{2}^{2}, \ldots, z_{d}^{2}\right) .
$$

The first factor on the right-hand side of (1.2) refers to an upsampled version of the data $d$.

Equation (1.2) can also be written using convolution with the so-called submasks of a. Let

$$
\mathrm{E}=\{0,1\}^{d}
$$

be the set of representatives of $\mathbb{Z}^{d} / 2 \mathbb{Z}^{d}$, given by the vertices of the unit cube $[0,1]^{d}$, containing

$$
\mathbf{0}=(0,0, \ldots, 0) \text { and } \mathbf{1}=(1,1, \ldots, 1) .
$$

Then, the $2^{d}$ submasks $\mathrm{a}_{\boldsymbol{e}}$ and their symbols $\mathrm{a}_{\boldsymbol{e}}(\boldsymbol{z})$ are defined by

$$
\mathrm{a}_{\boldsymbol{e}}=\left(\mathrm{a}_{\boldsymbol{e}+2 \boldsymbol{\alpha}}\right)_{\boldsymbol{\alpha} \in \mathbb{Z}^{d}} \text { and } \mathrm{a}_{\boldsymbol{e}}(\boldsymbol{z})=\sum_{\boldsymbol{\alpha} \in \mathbb{Z}^{d}} \mathrm{a}_{\boldsymbol{e}+2 \boldsymbol{\alpha}} \boldsymbol{z}^{\boldsymbol{\alpha}}, \quad \boldsymbol{e} \in \mathrm{E} .
$$

The standard decomposition

$$
\mathrm{a}(\boldsymbol{z})=\sum_{\boldsymbol{e} \in \mathrm{E}} \boldsymbol{z}^{e} \mathrm{a}_{\boldsymbol{e}}\left(\boldsymbol{z}^{2}\right)
$$

yields the equivalent form of the identity (1.2)

$$
\left(\mathcal{S}_{\mathrm{a}} \mathrm{d}\right)(\boldsymbol{z})=\sum_{\boldsymbol{e} \in \mathrm{E}} \boldsymbol{z}^{\boldsymbol{e}} \mathrm{d}\left(\boldsymbol{z}^{2}\right) \mathrm{a}_{\boldsymbol{e}}\left(\boldsymbol{z}^{2}\right) .
$$

This shows that a subdivision step is the result of convolving the input data $\mathrm{d}$ with each submask $a_{\boldsymbol{e}}$, which is a process of low pass filtering, followed by an interleaving process, i.e., upsampling and multiplication by $\boldsymbol{z}^{e}$, to produce the output data $\mathcal{S}_{\mathrm{a}}$ d.

We say that the subdivision scheme $\mathcal{S}_{a}$ is convergent, if for any starting sequence $\mathrm{d} \in \ell_{\infty}\left(\mathbb{Z}^{d}\right)$, there exists a uniformly continuous function $f_{\mathrm{d}}$ such that

$$
\lim _{r \rightarrow \infty} \sup _{\boldsymbol{\alpha} \in \mathbb{Z}^{d}}\left|\left(\mathcal{S}_{a}^{r} \mathrm{~d}\right)_{\boldsymbol{\alpha}}-f_{\mathrm{d}}\left(2^{-r} \boldsymbol{\alpha}\right)\right|=0,
$$

and $f_{\mathrm{d}} \neq 0$ for some initial data $\mathrm{d}$. This is the notion of $C$-convergence, also referred to as uniform convergence, see [4], where $L_{p}$-convergence for $1 \leq p<\infty$ is also discussed. The necessary condition for these types of convergence is now known to be the so-called sum rule of order 1 referring to the submasks

$$
\mathrm{a}_{\boldsymbol{e}}(\mathbf{1})=\sum_{\boldsymbol{\alpha} \in \mathbb{Z}^{d}} \mathrm{a}_{\boldsymbol{e}+2 \boldsymbol{\alpha}}=1, \quad \boldsymbol{e} \in \mathrm{E},
$$

see [4, Proposition 2.1] and [24, Theorem 3.1]. 
Another notion of convergence is used in the literature on multiresolution methods devoted to wavelet and frame constructions, for details see 7, Chapter 13], [13, Chapter 7], or [32, Chapter 6]. There, the convergence is characterized by the properties of the infinite product

$$
\prod_{j=0}^{\infty} \mathrm{a}^{\#}\left(\boldsymbol{z}^{2^{j}}\right), \quad \mathrm{a}^{\#}(\boldsymbol{z})=\frac{1}{2^{d}} \mathrm{a}(\boldsymbol{z})
$$

if $\boldsymbol{z}$ is restricted to the $d$-dimensional torus. To state some of the properties we switch to the real variables $\boldsymbol{\xi}=\left(\xi_{1}, \ldots, \xi_{d}\right)$ via the transformation $z_{j}=e^{-i \pi \xi_{j}}$, $j=1, \ldots, d$. The set (1.3) then transforms into the set

$$
\mathrm{Z}=\mathrm{Z}_{\mathrm{E}}=\left\{\varepsilon=e^{-i \pi e}: e \in \mathrm{E}\right\}=\{-1,+1\}^{d}
$$

of the vertices of the cube $[-1,+1]^{d}$, and the necessary condition (1.4) takes the equivalent form

$$
\mathrm{a}(\mathbf{1})=2^{d} \quad \text { and } \quad \mathrm{a}(\boldsymbol{\varepsilon})=0 \quad \text { for } \varepsilon \in \mathrm{Z}^{\prime}=\mathrm{Z} \backslash\{\mathbf{1}\} .
$$

For this reason we call $\mathrm{Z}^{\prime}$ the zero set, and the conditions in (1.6) the zero condition of order one (Condition $Z_{1}$ ). In the literature, both the conditions in (1.4) and their equivalent form in (1.6) are called the sum rules of order one.

More generally, we also use the higher order sum rules following the notation introduced and discussed in the survey paper [25], see also the references therein: The mask symbol a $(\boldsymbol{z})$ is said to satisfy the zero condition of order $k$ (Condition $Z_{k}$ ), if

$$
\mathrm{a}(\mathbf{1})=2^{d} \quad \text { and } \quad\left(D^{\mathbf{j}} \mathrm{a}\right)(\boldsymbol{\varepsilon})=0 \quad \text { for } \quad \boldsymbol{\varepsilon} \in \mathrm{Z}^{\prime}=\mathrm{Z} \backslash\{\mathbf{1}\} \quad \text { and } \quad|\mathbf{j}|<k .
$$

\section{Zero condition and the associated ideal}

In this section we show that condition $\mathrm{Z}_{1}$ fully determines the structure of the symbol a $(\boldsymbol{z})$ of any convergent scalar subdivision scheme, see Theorem A. The statement of this theorem is therefore the first step toward the desired multivariate generalization of the Factorization Property of univariate schemes. Theorem A is also crucial for understanding the structure of the symbol a $(\boldsymbol{z})$ satisfying condition $\mathrm{Z}_{k}$ for $k>1$. It also shows that the Factorization Property is replaced in the multivariate case by the decomposition (2.2).

The generators $q_{\Theta}$ in (2.2) are the symbols of subdivision schemes whose limit functions $1_{\Theta}$ are the characteristic functions of the parallelepiped spanned by the column vectors of certain matrices $\boldsymbol{\Theta}$. These functions are box splines of degree zero. In order to control the size of the mask, it is, thus, of importance to choose these box spline symbols appropriately, and to work in (2.2) with as few summands as possible. Theorem 2.6 shows how to meet these requirements.

A modification of the generators $q_{\Theta}$ is given in Theorem 2.11 and Theorem $\widetilde{A}$, at the end of this section. The modified generators $\widetilde{q}_{\Theta}$ have a useful algebraic property: they factor into $d$ linear polynomials. 
Since all convergent subdivision schemes satisfy condition $\mathrm{Z}_{1}$, we start with a characterization of the polynomial ideal

$$
\mathcal{I}=\left\{p \in \Pi^{d}: p(\varepsilon)=0 \text { for } \varepsilon \in \mathrm{Z}^{\prime}\right\}
$$

and later, in Section 3, of its powers

$$
\mathcal{I}^{k}=\left\{p \in \Pi^{d}:\left(D^{\mathbf{j}} p\right)(\varepsilon)=0 \text { for } \varepsilon \in \mathrm{Z}^{\prime},|\mathbf{j}|<k\right\}
$$

for $k>1$. The main result of this section, whose proof is a consequence of Theorem [2.6, states the following:

Theorem A. The mask symbol of any convergent d-variate subdivision scheme $\mathcal{S}_{\mathrm{a}}$ can be written in the form

$$
\mathrm{a}(\boldsymbol{z})=\sum_{\Theta} \lambda_{\Theta} \sigma_{\Theta}(\boldsymbol{z}) 2^{d} q_{\Theta}(\boldsymbol{z}) .
$$

The sum runs over all unimodular $d \times d$-submatrices $\Theta$ of $\left(\boldsymbol{X}_{d}^{(1)} \boldsymbol{X}_{d}^{(2)}\right)$ from (2.6). The polynomials $q_{\boldsymbol{\Theta}}(\boldsymbol{z})$ are defined in (2.7), $\sigma_{\boldsymbol{\Theta}}(\boldsymbol{z})$ are Laurent polynomials satisfying $\sigma_{\Theta}(\mathbf{1})=1$, and $\lambda_{\Theta}$ are real numbers subject to $\sum_{\Theta} \lambda_{\Theta}=1$.

Remark 2.1. Since the $2^{d} q_{\Theta}(\boldsymbol{z})$ are the mask symbols of certain box splines of degree zero, the mask $a$ is an affine combination of masks each of which originates from such a box spline convolved with some (smoothing) factor. Thus, Theorem A explains why such affine combinations were successfully studied before, see e.g., [9, 10, 11] for examples of bivariate and univariate schemes.

From the point of view of algebraic geometry, Theorem A tells us that the system of box spline symbols $q_{\Theta}$ in the representation (2.2) generates the ideal $\mathcal{I}$. This connects our work to the papers [30, 31] where the author has studied these ideals in detail and has characterized the ideal $\mathcal{I}$ in [30, Proposition 4.1] using the generators

$$
\mathcal{I}=<z_{1}^{2}-1, z_{2}^{2}-1, \ldots, z_{d}^{2}-1,\left(z_{1}+1\right)\left(z_{2}+1\right) \ldots\left(z_{d}+1\right)>.
$$

The characterization (2.3) is a special case of his more general results, see also [28, Example 4]. It also follows from the fact that the polynomial ideal

$$
\mathcal{J}:=\left\{p \in \Pi^{d}: p(\varepsilon)=0 \text { for } \varepsilon \in \mathrm{Z}\right\}
$$

of polynomials vanishing on $Z$ is generated by the polynomials

$$
z_{1}^{2}-1, z_{2}^{2}-1, \ldots, z_{d}^{2}-1
$$

This result is stated in [4, Lemma 2.3] with an elementary and constructive proof. Consequently, in order to determine a set of generators for the quotient ideal $\mathcal{I}$, it suffices to add to the generators of $\mathcal{J}$ the polynomial

$$
\left(z_{1}+1\right)\left(z_{2}+1\right) \ldots\left(z_{d}+1\right)
$$




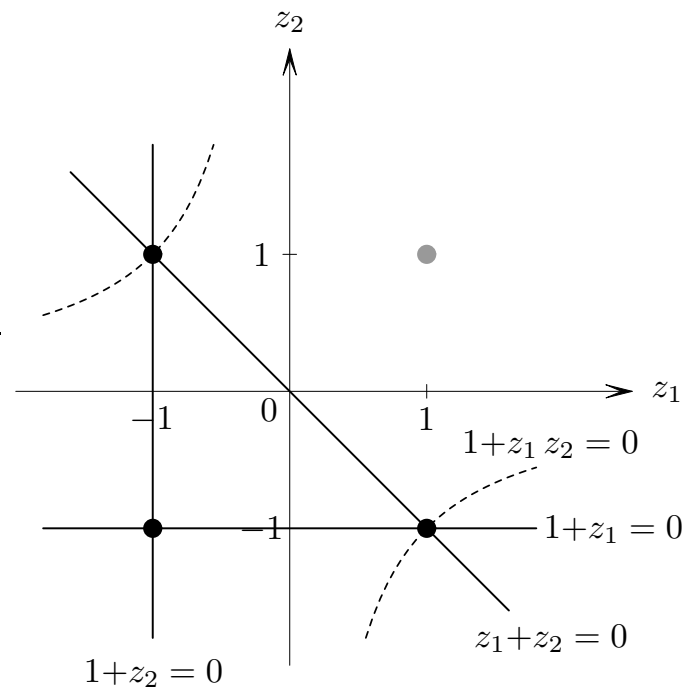

Figure 1: The ideal $\mathcal{I}$ from the algebraic geometer's point of view.

that vanishes on $\mathrm{Z}^{\prime}$, but does not vanish at $\mathbf{1}$.

In the two-dimensional case, guided by ideas from algebraic geometry, it seems natural to use the straight lines through the three points of $Z^{\prime}$, as shown in Figure 1, in order to find other generators for the ideal $\mathcal{I}$. This yields the following result.

Proposition 2.2. For $d=2$, we have

$$
\mathcal{I}=<\frac{1+z_{1}}{2} \frac{1+z_{2}}{2}, \frac{1+z_{1}}{2} \frac{z_{1}+z_{2}}{2}, \frac{1+z_{2}}{2} \frac{z_{1}+z_{2}}{2}>\text {. }
$$

Proof. By (2.3), we have for $d=2$

$$
\mathcal{I}=<z_{1}^{2}-1, z_{2}^{2}-1,\left(z_{1}+1\right)\left(z_{2}+1\right)>.
$$

Let us denote

$$
\widetilde{\mathcal{I}}=<\frac{1+z_{1}}{2} \frac{1+z_{2}}{2}, \frac{1+z_{1}}{2} \frac{z_{1}+z_{2}}{2}, \frac{1+z_{2}}{2} \frac{z_{1}+z_{2}}{2}>,
$$

then it suffices to show that the generators for $\mathcal{I}$ are contained in $\widetilde{\mathcal{I}}$ and vice versa.

To this end, note first that

$$
\begin{aligned}
z_{1}^{2}-1 & =-4 \frac{1+z_{1}}{2} \frac{1+z_{2}}{2}+4 \frac{1+z_{1}}{2} \frac{z_{1}+z_{2}}{2} \\
z_{2}^{2}-1 & =-4 \frac{1+z_{1}}{2} \frac{1+z_{2}}{2}+4 \frac{1+z_{2}}{2} \frac{z_{1}+z_{2}}{2} \\
\left(z_{1}+1\right)\left(z_{2}+1\right) & =4 \frac{1+z_{1}}{2} \frac{1+z_{2}}{2}
\end{aligned}
$$


and conversely that

$$
\begin{aligned}
& \frac{1+z_{1}}{2} \frac{1+z_{2}}{2}=\frac{1}{4}\left(z_{1}+1\right)\left(z_{2}+1\right) \\
& \frac{1+z_{1}}{2} \frac{z_{1}+z_{2}}{2}=\frac{1}{4}\left(z_{1}^{2}-1\right)+\frac{1}{4}\left(z_{1}+1\right)\left(z_{2}+1\right) \\
& \frac{1+z_{2}}{2} \frac{z_{1}+z_{2}}{2}=\frac{1}{4}\left(z_{2}^{2}-1\right)+\frac{1}{4}\left(z_{1}+1\right)\left(z_{2}+1\right) .
\end{aligned}
$$

This shows that $\widetilde{\mathcal{I}}=\mathcal{I}$ as claimed.

Remark 2.3. The three functions in (2.4) are remarkably close to the symbols of the three box splines of degree zero on the three-directional grid. Indeed, if we reflect the standard three-directional grid about one of the coordinate axes, $i$.e., use the grid spanned by the three vectors $\left(\begin{array}{l}1 \\ 0\end{array}\right),\left(\begin{array}{l}0 \\ 1\end{array}\right)$, and $\left(\begin{array}{c}1 \\ -1\end{array}\right)$, then the box splines of degree zero have the mask symbols

$$
4 \frac{1+z_{1}}{2} \frac{1+z_{2}}{2}, \quad 4 \frac{1+z_{1}}{2} \frac{1+z_{1} / z_{2}}{2}, \quad 4 \frac{1+z_{2}}{2} \frac{1+z_{1} / z_{2}}{2} .
$$

Since we can write $z_{1}+z_{2}=z_{2}\left(1+z_{1} / z_{2}\right)$, these are just the functions in (2.4) up to an appropriate normalization.

It follows that the result of Proposition 2.2 can as well use the usual box spline symbols: The reflection of the three-directional grid about the $z_{1}$-axis, as mentioned before, corresponds to the variable transformation $\left(z_{1}, z_{2}\right) \mapsto$ $\left(z_{1}, 1 / z_{2}\right)$, and then (2.4) becomes - again after a proper normalization-

$$
\mathcal{I}=<\frac{1+z_{1}}{2} \frac{1+z_{2}}{2}, \frac{1+z_{1}}{2} \frac{1+z_{1} z_{2}}{2}, \frac{1+z_{2}}{2} \frac{1+z_{1} z_{2}}{2}>.
$$

From the algebraic geometer's point of view, this amounts to replacing the straight line $z_{1}+z_{2}=0$ in Figure 1 by the hyperbola $1+z_{1} z_{2}=0$.

The identity (2.5) is the statement of Theorem 2.6 for the case $d=2$. To be able to state this result in general, we first need to provide some additional notation. As before, we denote by $\mathrm{E}=\{0,1\}^{d}$ the set of vertices of the $d$ dimensional hypercube, and let $\mathrm{E}^{\prime}=\mathrm{E} \backslash\{\mathbf{0}\}$. Collecting the elements of $\mathrm{E}^{\prime}$ in the matrix $\boldsymbol{X}_{d}=(\boldsymbol{e})_{\boldsymbol{e} \in \mathrm{E}^{\prime}}$ yields

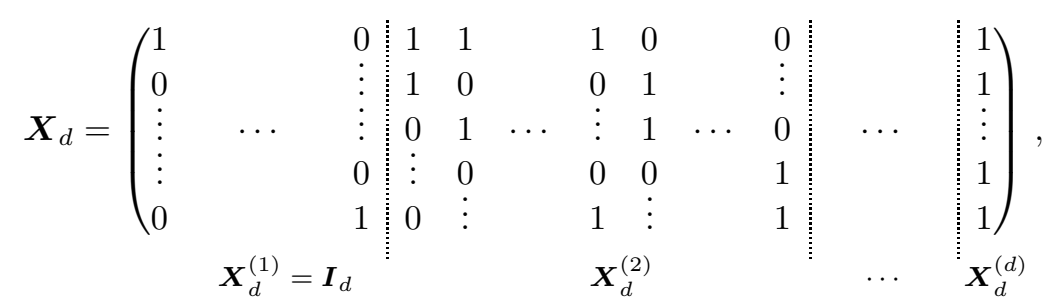

where each column of the submatrix $\boldsymbol{X}_{d}^{(k)}$ contains exactly $k$ entries equal to 1 . Thus $\boldsymbol{X}_{d}^{(1)}$ consists of the standard unit vectors $\boldsymbol{e}_{k}, k=1, \ldots, d$, while $\boldsymbol{X}_{d}^{(2)}$ 
contains the vectors $\boldsymbol{e}_{j}+\boldsymbol{e}_{k}, j \neq k$, etc. We treat the columns of $\boldsymbol{X}_{d}$ as directional vectors from which we build the box splines of degree zero, i. e., the characteristic functions of certain parallelepipeds. This means that we take any $d$ columns from $\boldsymbol{X}_{d}$ to produce a square submatrix $\boldsymbol{\Theta}$ of $\boldsymbol{X}_{d}$. With each such $\Theta$, we associate the normalized polynomial

$$
q_{\Theta}(\boldsymbol{z})=\prod_{\boldsymbol{\theta} \in \boldsymbol{\Theta}} \frac{1+\boldsymbol{z}^{\boldsymbol{\theta}}}{2},
$$

where $\boldsymbol{\theta}$ runs through the columns of $\boldsymbol{\Theta}$, with $q_{\boldsymbol{\Theta}}(\mathbf{1})=1$.

Proposition 2.4. For any $d \times d$-submatrix $\boldsymbol{\Theta}$ of $\boldsymbol{X}_{d}$ in (2.6), we have

$$
q_{\Theta} \in \mathcal{I} \quad \Longleftrightarrow \quad \operatorname{det} \Theta \equiv 1(\bmod 2) \text {. }
$$

Proof. By definition, $q_{\Theta} \in \mathcal{I}$ if and only if

$$
\forall \varepsilon \in \mathrm{Z}^{\prime}: \quad q_{\Theta}(\varepsilon)=\prod_{\boldsymbol{\theta} \in \boldsymbol{\Theta}} \frac{1+\varepsilon^{\boldsymbol{\theta}}}{2}=0 .
$$

Due to $\boldsymbol{\varepsilon}^{\boldsymbol{\theta}}=e^{-i \pi \boldsymbol{e}^{T} \boldsymbol{\theta}}, \boldsymbol{\varepsilon} \in \mathrm{Z}^{\prime}$, this is equivalent to

$$
\forall \boldsymbol{e} \in \mathrm{E}^{\prime}: \exists \boldsymbol{\theta} \in \boldsymbol{\Theta}: \quad \boldsymbol{e}^{T} \boldsymbol{\theta} \equiv 1(\bmod 2) .
$$

I. e., the map

$$
L: \mathbb{Z}^{d} \rightarrow \mathbb{Z}^{d}, \quad \boldsymbol{x}^{T} \mapsto \boldsymbol{x}^{T} \boldsymbol{\Theta},
$$

has the property

$$
\forall \boldsymbol{e} \in \mathrm{E}^{\prime}: \quad L\left(\boldsymbol{e}^{T}\right) \not \equiv \mathbf{0}^{T}(\bmod 2) .
$$

As we are only interested in the parity of $\operatorname{det} \boldsymbol{\Theta}$, we may employ the ring homomorphism $\mathbb{Z} \rightarrow \mathbb{Z}_{2}=\mathbb{Z} / 2 \mathbb{Z}$ mapping even and odd numbers to their coset representatives $\overline{0}$ and $\overline{1}$, respectively. It extends naturally to a matrix ring homomorphism $\mathbb{Z}^{d \times d} \rightarrow\left(\mathbb{Z}_{2}\right)^{d \times d}$ and thus induces a linear map $\bar{L}:\left(\mathbb{Z}_{2}\right)^{d} \rightarrow$ $\left(\mathbb{Z}_{2}\right)^{d}$ satisfying $\operatorname{det} \bar{L}=\overline{\operatorname{det} L}$. Since the set $\mathrm{E}$ is a complete set of coset representatives of the vector space $\left(\mathbb{Z}_{2}\right)^{d}=\mathbb{Z}^{d} / 2 \mathbb{Z}^{d}$, property (2.8) is equivalent to

$$
\operatorname{ker}(\bar{L})=\left\{\overline{\mathbf{0}}^{T}\right\},
$$

i.e., $\bar{L}$ is a vector space automorphism and thus

$$
\operatorname{det} \bar{L} \neq \overline{0} \quad \text { in } \mathbb{Z}_{2} .
$$

In other words,

$$
\operatorname{det} \boldsymbol{\Theta}=\operatorname{det} L \not \equiv 0(\bmod 2),
$$

which yields the claim.

The next result, which is important for the proof of Theorem A, states that, conversely, the ideal $\mathcal{I}$ is generated by a subfamily of the elements $q_{\Theta}$. It is worth noting that the condition $\operatorname{det} \boldsymbol{\Theta}= \pm 1$ is equivalent to $\boldsymbol{\Theta}$ having an integer inverse and, thus, to the fact that its columns generate the integer grid $\mathbb{Z}^{d}$. 
Lemma 2.5. For $k=1, \ldots, d$, we have

$z_{k}^{2}-1 \in<\left\{q_{\boldsymbol{\Theta}}: \boldsymbol{\Theta} d \times d\right.$-submatrix of $\left.\left(\boldsymbol{X}_{d}^{(1)}: \boldsymbol{X}_{d}^{(2)}\right), \operatorname{det} \boldsymbol{\Theta}= \pm 1, \boldsymbol{e}_{k} \in \boldsymbol{\Theta}\right\}>$.

Proof. For $d=1$, the lemma simply claims that

$$
z^{2}-1 \in<\frac{1+z}{2}>,
$$

which follows from $z^{2}-1=2(z-1) \frac{1+z}{2}$.

For $d \geq 2$, it suffices to prove that

$$
z_{k}-1 \in<\left\{q_{\Theta}: \Theta \in \mathcal{U}_{d}^{(k)}\right\}>\text { for } k=1, \ldots, d,
$$

where the family $\mathcal{U}_{d}^{(k)}$ is defined to be

$$
\mathcal{U}_{d}^{(k)}=\left\{\boldsymbol{\Theta}: d \times(d-1) \text {-submatrix of }\left(\boldsymbol{X}_{d}^{(1)} \boldsymbol{X}_{d}^{(2)}\right), \operatorname{det}\left(\boldsymbol{\Theta}: \boldsymbol{e}_{k}\right)= \pm 1\right\} .
$$

The proof is by induction on $d$. For $d=2$, we have $\left(\boldsymbol{X}_{d}^{(1)} \boldsymbol{X}_{d}^{(2)}\right)=\left(\begin{array}{lll}1 & 0 & 1 \\ 0 & 1 & 1\end{array}\right)$. From this, we find for $k=1$

$$
\mathcal{U}_{2}^{(1)}=\left\{\left(\begin{array}{l}
0 \\
1
\end{array}\right),\left(\begin{array}{l}
1 \\
1
\end{array}\right)\right\}
$$

and thus the lemma claims that

$$
z_{1}-1 \in<\frac{1+z_{2}}{2}, \frac{1+z_{1} z_{2}}{2}>
$$

which follows from

$$
z_{1}-1=2 z_{1} \frac{1+z_{2}}{2}-2 \frac{1+z_{1} z_{2}}{2} .
$$

The claim for $k=2$ follows by symmetry.

For the induction step, we consider first $k=d+1$ and write

$$
z_{d+1}-1=-z_{d+1}\left(z_{d}-1\right) \frac{1+z_{d}}{2}+\left(z_{d}-1\right) \frac{1+z_{d} z_{d+1}}{2}+\left(z_{d+1}-1\right) \frac{1+z_{d}}{2} .
$$

By the induction hypothesis, we can write

$$
z_{d}-1=\sum_{\boldsymbol{\Theta} \in \mathcal{U}_{d}^{(d)}} p_{\boldsymbol{\Theta}}\left(z_{1}, \ldots, z_{d}\right) q_{\boldsymbol{\Theta}}\left(z_{1}, \ldots, z_{d}\right)
$$

for certain polynomials $p_{\Theta}$. For $\boldsymbol{\Theta} \in \mathcal{U}_{d}^{(d)}$, we define

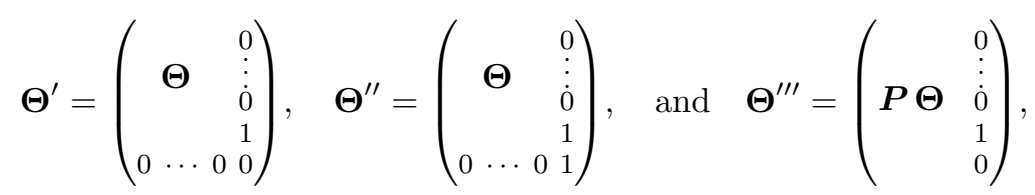


where

$$
\boldsymbol{P}=\left(\begin{array}{cccc}
1 & & 0 & 0 \\
& \ddots & & \vdots \\
0 & & 1 & 0 \\
0 & \cdots & 0 & 0 \\
0 & \cdots & 0 & 1
\end{array}\right)
$$

It is easily seen that this yields $\boldsymbol{\Theta}^{\prime}, \boldsymbol{\Theta}^{\prime \prime}, \boldsymbol{\Theta}^{\prime \prime \prime} \in \mathcal{U}_{d+1}^{(d+1)}$ with

$$
\begin{aligned}
q_{\boldsymbol{\Theta}^{\prime}}\left(z_{1}, \ldots, z_{d+1}\right) & =q_{\boldsymbol{\Theta}}\left(z_{1}, \ldots, z_{d}\right) \frac{1+z_{d}}{2}, \\
q_{\boldsymbol{\Theta}^{\prime \prime}}\left(z_{1}, \ldots, z_{d+1}\right) & =q_{\boldsymbol{\Theta}}\left(z_{1}, \ldots, z_{d}\right) \frac{1+z_{d} z_{d+1}}{2}, \\
\text { and } \quad q_{\boldsymbol{\Theta}^{\prime \prime \prime}}\left(z_{1}, \ldots, z_{d+1}\right) & =q_{\boldsymbol{\Theta}}\left(z_{1}, \ldots, z_{d-1}, z_{d+1}\right) \frac{1+z_{d}}{2} .
\end{aligned}
$$

Using these identities, we obtain from (2.11)

$$
\begin{aligned}
-z_{d+1}\left(z_{d}-1\right) \frac{1+z_{d}}{2} & =\sum_{\boldsymbol{\Theta} \in \mathcal{U}_{d}^{(d)}}-z_{d+1} p_{\boldsymbol{\Theta}}\left(z_{1}, \ldots, z_{d}\right) q_{\boldsymbol{\Theta}^{\prime}}\left(z_{1}, \ldots, z_{d+1}\right), \\
\left(z_{d}-1\right) \frac{1+z_{d} z_{d+1}}{2} & =\sum_{\boldsymbol{\Theta} \in \mathcal{U}_{d}^{(d)}} p_{\boldsymbol{\Theta}}\left(z_{1}, \ldots, z_{d}\right) q_{\boldsymbol{\Theta}^{\prime \prime}}\left(z_{1}, \ldots, z_{d+1}\right),
\end{aligned}
$$

and, by replacing $z_{d}$ by $z_{d+1}$ in (2.11),

$$
\left(z_{d+1}-1\right) \frac{1+z_{d}}{2}=\sum_{\boldsymbol{\Theta} \in \mathcal{U}_{d}^{(d)}} p_{\Theta}\left(z_{1}, \ldots, z_{d-1}, z_{d+1}\right) q_{\Theta^{\prime \prime \prime}}\left(z_{1}, \ldots, z_{d+1}\right) .
$$

Substituting these three identities into (2.10) yields a representation for $z_{d+1}-1$ of the desired form. A corresponding representation for $z_{k}-1$ with $k \leq d$ can be obtained by a cyclic permutation of the indices.

This completes the induction.

We would like to emphasize again that a very important consequence of the following theorem, for subdivision schemes, is stated as the main result of this section in Theorem A.

Theorem 2.6. The ideal $\mathcal{I}$ is generated by the elements $q_{\boldsymbol{\Theta}}$, where $\boldsymbol{\Theta}$ are the unimodular $d \times d$-submatrices of $\left(\boldsymbol{X}_{d}^{(1)}: \boldsymbol{X}_{d}^{(2)}\right)$ from (2.6).

Proof. One of the inclusions follows from Proposition 2.4. In the light of (2.3), the other inclusion is an immediate consequence of Lemma 2.5 and the fact that

$$
\left(z_{1}+1\right)\left(z_{2}+1\right) \ldots\left(z_{d}+1\right)=2^{d} q_{\boldsymbol{\Theta}}(\boldsymbol{z}) \text { for } \quad \boldsymbol{\Theta}=\boldsymbol{I}_{d}=\boldsymbol{X}_{d}^{(1)} .
$$

It is worth noting that the condition $\boldsymbol{e}_{k} \in \boldsymbol{\Theta}$ in Lemma 2.5 is equivalent to the divisibility of $q_{\Theta}$ by $1+z_{k}$, which seems natural since $1+z_{k}$ obviously divides $1-z_{k}^{2}$. Furthermore, the fact that each matrix $\Theta$ in Theorem 2.6 contains a standard unit vector as its column is a consequence of the following result. 
Lemma 2.7. Any $d \times d$-submatrix $\boldsymbol{\Theta}$ of $\boldsymbol{X}_{d}^{(2)}$ satisfies

$$
\operatorname{det} \boldsymbol{\Theta} \equiv 0(\bmod 2) .
$$

Proof. A $d \times d$-submatrix $\boldsymbol{\Theta}$ of $\boldsymbol{X}_{d}^{(2)}$ contains in each column exactly two entries equal to one (and the others are equal to zero). For the linear operator $L$ introduced in the proof of Proposition 2.4, this implies

$$
L\left(\mathbf{1}^{T}\right)=\mathbf{1}^{T} \boldsymbol{\Theta}=2 \cdot \mathbf{1}^{T} \equiv \mathbf{0}^{T}(\bmod 2)
$$

i.e., $\mathbf{1}^{T} \in \operatorname{ker}(\bar{L})$. From this, we may conclude that $\operatorname{det} \bar{L}=\overline{0}$ in $\mathbb{Z}_{2}$ and, thus, $\operatorname{det} \boldsymbol{\Theta}=\operatorname{det} L \equiv 0(\bmod 2)$.

Examples 2.8. We list the generators $q_{\Theta}$ of $\mathcal{I}$ for low-dimensional cases.

- For $d=1$, we have $\boldsymbol{X}_{1}=(1)$, so the only submatrix is $\boldsymbol{\Theta}=\boldsymbol{X}_{1}$ with

$$
q_{\Theta}(z)=\frac{1+z}{2},
$$

and $\mathcal{I}$ is the principal ideal generated by this function.

- For $d=2$, we have

$$
\boldsymbol{X}_{2}=\left(\begin{array}{ll:l}
1 & 0 & 1 \\
0 & 1 & 1
\end{array}\right)
$$

which yields the following three generators for $\mathcal{I}$ :

$$
\begin{aligned}
& \boldsymbol{\Theta}_{1}=\left(\begin{array}{ll}
1 & 0 \\
0 & 1
\end{array}\right) \quad \text { with } \quad q_{\boldsymbol{\Theta}_{1}}(z)=\frac{1+z_{1}}{2} \frac{1+z_{2}}{2}, \\
& \boldsymbol{\Theta}_{2}=\left(\begin{array}{ll}
1 & 1 \\
0 & 1
\end{array}\right) \quad \text { with } \quad q_{\Theta_{2}}(z)=\frac{1+z_{1}}{2} \frac{1+z_{1} z_{2}}{2}, \\
& \boldsymbol{\Theta}_{3}=\left(\begin{array}{ll}
0 & 1 \\
1 & 1
\end{array}\right) \quad \text { with } \quad q_{\Theta_{3}}(z)=\frac{1+z_{2}}{2} \frac{1+z_{1} z_{2}}{2},
\end{aligned}
$$

as stated in (2.5). These three functions generate $\mathcal{I}$ minimally in the sense that no two of them generate all of $\mathcal{I}$.

- In the case $d=3$, we have

$$
\boldsymbol{X}_{3}=\left(\begin{array}{lll:lll:l}
1 & 0 & 0 & 1 & 1 & 0 & 1 \\
0 & 1 & 0 & 1 & 0 & 1 & 1 \\
0 & 0 & 1 & 0 & 1 & 1 & 1
\end{array}\right)
$$

The submatrices $\Theta$ with even determinants are obtained by selecting the columns $(1,2,4),(1,3,5),(1,6,7),(2,3,6),(2,5,7)$, and $(3,4,7)$, $\operatorname{det} \boldsymbol{\Theta}=0$, and $(4,5,6)$, $\operatorname{det} \boldsymbol{\Theta}=-2$ (compare Lemma 2.7). The remaining $\left(\begin{array}{l}7 \\ 3\end{array}\right)-7=28$ submatrices have determinant \pm 1 and thus describe elements of $\mathcal{I}$. Restricting ourselves to $\left(\boldsymbol{X}_{d}^{(1)} \boldsymbol{X}_{d}^{(2)}\right)$ as stated in Theorem 2.6 yields $\left(\begin{array}{l}6 \\ 3\end{array}\right)-4=16$ elements generating the ideal $\mathcal{I}$. However, this system is highly redundant: using an algebraic manipulation program, we found that it contains 64 subsets of 10 elements each which generate $\mathcal{I}$ minimally (in the sense that in each case, omitting any one of the 10 elements does not yield a set of generators anymore). 


\section{Geometric interpretation and modification.}

The columns of $\boldsymbol{X}_{d}$ describe the edges and the various diagonals of the $d$ hypercube. By Proposition 2.4 the polynomial $q_{\Theta}$ is an element of $\mathcal{I}$ if and only if the columns of $\Theta$ span a parallelepiped with an odd $d$-volume. Theorem 2.6 states that in order to generate $\mathcal{I}$, it suffices to restrict ourselves to the edges $\boldsymbol{e}_{k}$ and the 2-surface-diagonals $\boldsymbol{e}_{j}+\boldsymbol{e}_{k}, j \neq k$, of the hypercube only, and to consider only parallelepipeds with $d$-volume equal to one. Lemma 2.7 shows that these parallelepipeds all have at least one unit vector as an edge.

Proposition 2.2 leads to an alternative approach. For $d=2$, the three vectors $\boldsymbol{e}_{1}=\left(\begin{array}{l}1 \\ 0\end{array}\right), \boldsymbol{e}_{2}=\left(\begin{array}{l}0 \\ 1\end{array}\right)$, and $\boldsymbol{e}_{1}-\boldsymbol{e}_{2}=\left(\begin{array}{c}1 \\ -1\end{array}\right)$ can also be seen as the edges of the 2 -simplex. Since for $d>2$ we only need to use the diagonals of the 2dimensional surfaces of the $d$-hypercube, it seems natural to consider the edges of the $d$-simplex instead.

To this end, we describe a different family of polynomials. For any $d \times d$ submatrix $\boldsymbol{\Theta}$ of $\left(\boldsymbol{X}_{d}^{(1)} \boldsymbol{X}_{d}^{(2)}\right)$, define

$$
\widetilde{q}_{\boldsymbol{\Theta}}(\boldsymbol{z})=\prod_{\boldsymbol{\theta} \in \boldsymbol{\Theta}} \widetilde{r}_{\boldsymbol{\theta}}(\boldsymbol{z}) \quad \text { with } \quad \widetilde{r}_{\boldsymbol{\theta}}(\boldsymbol{z})= \begin{cases}\frac{1+z_{k}}{2}, & \text { if } \boldsymbol{\theta}=\boldsymbol{e}_{k}, \\ \frac{z_{j}+z_{k}}{2}, & \text { if } \boldsymbol{\theta}=\boldsymbol{e}_{j}+\boldsymbol{e}_{k} .\end{cases}
$$

Geometrically, this amounts for $d=2$ to replacing the vector $\left(\begin{array}{l}1 \\ 1\end{array}\right)$ by $\left(\begin{array}{c}1 \\ -1\end{array}\right)$ as third direction in the three-directional grid. Equivalently, this can be seen as a reflection of the grid about one of the coordinate axes (compare Remark 2.3).

Algebraically, this has the advantage that each factor $\widetilde{r}_{\boldsymbol{\theta}}$ is linear and therefore that all the $\widetilde{q}_{\Theta}$ share the same total degree $d$. These functions still satisfy the analogues of Proposition 2.4. Lemma 2.5. and, consequently, of Theorem 2.6 and Theorem A.

Proposition 2.9. For any $d \times d$-submatrix $\Theta$ of $\left(\boldsymbol{X}_{d}^{(1)} \boldsymbol{X}_{d}^{(2)}\right)$ in (2.6), we have

$$
\widetilde{q}_{\Theta} \in \mathcal{I} \Longleftrightarrow \operatorname{det} \Theta \equiv 1(\bmod 2) \text {. }
$$

Proof. For $\varepsilon=\left(\varepsilon_{1}, \ldots, \varepsilon_{d}\right) \in \mathrm{Z}^{\prime}=\{-1,+1\}^{d} \backslash\{\mathbf{1}\}$, we have

$$
\frac{\varepsilon_{j}+\varepsilon_{k}}{2}=\varepsilon_{k} \frac{1+\varepsilon_{j} / \varepsilon_{k}}{2}= \pm \frac{1+\varepsilon_{j} \varepsilon_{k}}{2}, \quad j, k=1, \ldots, d,
$$

and, therefore, $\widetilde{q}_{\Theta}(\varepsilon)= \pm q_{\Theta}(\varepsilon)$. Consequently,

$$
\left.\widetilde{q}_{\Theta} \in \mathcal{I} \Longleftrightarrow \widetilde{q}_{\Theta}\right|_{\mathrm{Z}^{\prime}}=\left.0 \Longleftrightarrow q_{\Theta}\right|_{\mathrm{Z}^{\prime}}=0 \Longleftrightarrow q_{\Theta} \in \mathcal{I} \text {. }
$$

The claim follows by Proposition 2.4 .

Lemma 2.10. For $k=1, \ldots, d$, we have

$$
1-z_{k}^{2} \in\left\langle\left\{\widetilde{q}_{\boldsymbol{\Theta}}: \boldsymbol{\Theta} \subseteq\left(\boldsymbol{X}_{d}^{(1)} \boldsymbol{X}_{d}^{(2)}\right), \operatorname{det} \boldsymbol{\Theta}= \pm 1, \boldsymbol{e}_{k} \in \boldsymbol{\Theta}\right\}>\right.
$$


Proof. The proof is mutatis mutandis the same as that of Lemma 2.5] except that (2.9) becomes

$$
z_{1}-1=-2 \frac{1+z_{2}}{2}+2 \frac{z_{1}+z_{2}}{2}
$$

and 2.10 becomes

$$
z_{d+1}-1=\left(z_{d}-1\right) \frac{1+z_{d}}{2}-\left(z_{d}-1\right) \frac{z_{d}+z_{d+1}}{2}+\left(z_{d+1}-1\right) \frac{1+z_{d}}{2} .
$$

Theorem 2.11. The ideal $\mathcal{I}$ is generated by the elements $\widetilde{q}_{\boldsymbol{\Theta}}$, where $\boldsymbol{\Theta}$ are the unimodular $d \times d$-submatrices of $\left(\boldsymbol{X}_{d}^{(1)}: \boldsymbol{X}_{d}^{(2)}\right)$ from (2.6).

Proof. As in the proof of Theorem 2.6, using Lemma 2.10 instead of Lemma 2.5]

The interpretation of Theorem 2.11 as a property of subdivision schemes leads to the following modification of Theorem A:

Theorem $\widetilde{\mathbf{A}}$. The mask symbol of any convergent d-variate subdivision scheme $\mathcal{S}_{\mathrm{a}}$ can be written in the form

$$
\mathrm{a}(\boldsymbol{z})=\sum_{\Theta} \lambda_{\Theta} \sigma_{\Theta}(\boldsymbol{z}) 2^{d} \widetilde{q}_{\Theta}(\boldsymbol{z})
$$

where $\sigma_{\Theta}(\boldsymbol{z})$ are Laurent polynomials satisfying $\sigma_{\Theta}(\mathbf{1})=1$, and $\lambda_{\Theta}$ are real numbers subject to $\sum_{\Theta} \lambda_{\Theta}=1$. The sum runs over all unimodular $d \times d$ submatrices $\boldsymbol{\Theta}$ of $\left(\boldsymbol{X}_{d}^{(1)} \boldsymbol{X}_{d}^{(2)}\right)$ from (2.6).

\section{Zero condition of higher orders and powers of $\mathcal{I}$ for $d=2$}

In this section, we describe families of generators for the ideals $\mathcal{I}^{k}$, for $k>1$, and the implications on the representation of mask symbols from Theorem A. The case $d=2$ is of special interest in subdivision. Therefore, most of this section is devoted to the study of this case, see in particular Theorem $\mathrm{C}$ below and the remarks at the end of Section 3.1.

We start with a simple observation from ideal theory, namely, the fact that the product $\mathcal{I}_{1} \cdot \mathcal{I}_{2}$ of two ideals $\mathcal{I}_{1}=\left\langle\left\{a_{j}: j=1, \ldots, n\right\}\right\rangle$ and $\mathcal{I}_{2}=\left\langle\left\{b_{k}: k=1, \ldots, m\right\}\right\rangle$ in a ring is generated by the pointwise products of the corresponding generating sets

$$
\mathcal{I}_{1} \cdot \mathcal{I}_{2}=<\left\{a_{j} b_{k}: j=1, \ldots, n, k=1, \ldots, m\right\}>\text {. }
$$

Applying this to the construction of generators for $\mathcal{I}^{k}$, we get the following immediate generalization of Theorem A. 
Theorem B. A convergent d-variate subdivision scheme $\mathcal{S}_{\mathrm{a}}$ satisfies the condition $\mathrm{Z}_{k}$ if and only if its mask symbol can be written in the form

$$
\mathrm{a}(\boldsymbol{z})=\sum_{j} \lambda_{j} \sigma_{j}(\boldsymbol{z}) 2^{d} Q_{j}(\boldsymbol{z}),
$$

where $Q_{j}(\boldsymbol{z})$ are $k$-fold products of Laurent polynomials $q_{\Theta}$ with unimodular $d \times d$-submatrices $\boldsymbol{\Theta}$ of $X_{d}$ from (2.6), $\sigma_{j}(\boldsymbol{z})$ are Laurent polynomials normalized by $\sigma_{j}(\mathbf{1})=1$, and $\lambda_{j}$ are real numbers subject to $\sum_{j} \lambda_{j}=1$.

Note that the normalizations assumed in Theorem B imply a $(\mathbf{1})=2^{d}$.

In the bivariate case, we can be much more specific and show that the generators for $\mathcal{I}^{k}$ are the mask symbols of certain three-directional box splines. The latter have been studied thoroughly in [2, 3, 8, 27], and their mask symbols have the form $B_{\alpha, \beta, \gamma}=4 B_{\alpha, \beta, \gamma}^{\#}$, where

$$
B_{\alpha, \beta, \gamma}^{\#}\left(z_{1}, z_{2}\right)=\left(\frac{1+z_{1}}{2}\right)^{\alpha}\left(\frac{1+z_{2}}{2}\right)^{\beta}\left(\frac{1+z_{1} z_{2}}{2}\right)^{\gamma}, \quad \alpha, \beta, \gamma \in \mathbb{N}_{0},
$$

are the normalized box spline symbols satisfying $B_{\alpha, \beta, \gamma}^{\#}(1,1)=1$. The three indices $\alpha, \beta, \gamma$ correspond to the multiplicities of the three vectors $\boldsymbol{e}_{1}, \boldsymbol{e}_{2}$ and $\boldsymbol{e}_{1}+\boldsymbol{e}_{2}$. In this notation, (2.5) becomes

$$
\mathcal{I}=<B_{1,1,0}^{\#}, B_{1,0,1}^{\#}, B_{0,1,1}^{\#}>.
$$

Note that the family of these (normalized) box spline symbols is partially ordered and closed under multiplication, since

$$
B_{\alpha, \beta, \gamma}^{\#} \cdot B_{\alpha^{\prime}, \beta^{\prime}, \gamma^{\prime}}^{\#}=B_{\alpha+\alpha^{\prime}, \beta+\beta^{\prime}, \gamma+\gamma^{\prime}}^{\#} .
$$

Furthermore, they satisfy the following relation that we need later on.

Lemma 3.1. For any given triple $(\alpha, \beta, \gamma) \in \mathbb{N}_{0}^{3}$, the ideal generated by the three symbols $B_{\alpha+1, \beta, \gamma}^{\#}, B_{\alpha, \beta+1, \gamma}^{\#}$, and $B_{\alpha, \beta, \gamma+1}^{\#}$ is the principal ideal generated by $B_{\alpha, \beta, \gamma}^{\#}$.

Proof. Since each of the symbols $B_{\alpha+1, \beta, \gamma}^{\#}, B_{\alpha, \beta+1, \gamma}^{\#}$, and $B_{\alpha, \beta, \gamma+1}^{\#}$ is a multiple of $B_{\alpha, \beta, \gamma}^{\#}$, we only have to show that the latter can be generated from the former three. To this end, we make use of the identity

$$
\frac{1}{2}\left(1-z_{2}\right) B_{1,0,0}^{\#}\left(z_{1}, z_{2}\right)+\frac{1}{2}\left(1-z_{1}\right) B_{0,1,0}^{\#}\left(z_{1}, z_{2}\right)+B_{0,0,1}^{\#}\left(z_{1}, z_{2}\right)=1 .
$$

Multiplying both sides by $B_{\alpha, \beta, \gamma}^{\#}$ proves the lemma.

In the light of (3.3), it is natural to expect that the higher powers of the ideal $\mathcal{I}$ are generated by box spline symbols of higher order, as the following result shows. 
Theorem 3.2. In the bivariate case, the $k$-th power $\mathcal{I}^{k}, k \in \mathbb{N}$, of the ideal $\mathcal{I}$ is generated by the set of three-directional box spline symbols

$$
\mathrm{I}_{k}:=\left\{B_{\beta, \beta, \alpha}^{\#}, B_{\beta, \alpha, \beta}^{\#}, B_{\alpha, \beta, \beta}^{\#}: \alpha=0,1, \ldots,\left\lfloor\frac{k}{2}\right\rfloor, \beta=k-\alpha\right\} .
$$

Proof. The proof is by induction on $k$. For $k=1$, the claim is just the identity (3.3).

For the induction step, we write $\mathcal{I}^{k+1}=\mathcal{I} \cdot \mathcal{I}^{k}$ and apply (3.1). Using once more (3.3) and the induction hypothesis $\mathcal{I}^{k}=\left\langle\mathrm{I}_{k}>\right.$ yields $\left.\mathcal{I}^{k+1}=<\mathrm{I}_{k+1}^{\prime}\right\rangle$ with

$\mathrm{I}_{k+1}^{\prime}=\left\{\begin{array}{l}B_{\beta+1, \beta+1, \alpha}^{\#}, B_{\beta+1, \alpha+1, \beta}^{\#}, B_{\alpha+1, \beta+1, \beta}^{\#}, \\ B_{\beta+1, \beta, \alpha+1}^{\#}, B_{\beta+1, \alpha, \beta+1}^{\#}, B_{\alpha+1, \beta, \beta+1}^{\#}, \alpha=0,1, \ldots,\left\lfloor\frac{k}{2}\right\rfloor, \beta=k-\alpha \\ B_{\beta, \beta+1, \alpha+1}^{\#}, B_{\beta, \alpha+1, \beta+1}^{\#}, B_{\alpha, \beta+1, \beta+1}^{\#},\end{array}\right\}$.

So we need to show that $\left\langle\mathrm{I}_{k+1}^{\prime}\right\rangle=\left\langle\mathrm{I}_{k+1}>\right.$ where

$$
\mathrm{I}_{k+1}=\left\{B_{\delta, \delta, \gamma}^{\#}, B_{\delta, \gamma, \delta}^{\#}, B_{\gamma, \delta, \delta}^{\#}: \gamma=0,1, \ldots,\left\lfloor\frac{k+1}{2}\right\rfloor, \delta=k+1-\gamma\right\} .
$$

To this end, it suffices to show that both $\mathrm{I}_{k+1} \subseteq<\mathrm{I}_{k+1}^{\prime}>$ and $\mathrm{I}_{k+1}^{\prime} \subseteq<\mathrm{I}_{k+1}>$.

Firstly, note that the elements of $\mathrm{I}_{k+1}$ are the diagonal elements in the list $\mathrm{I}_{k+1}^{\prime}$ with $\gamma=\alpha$ and thus $\delta=\beta+1$, with the only exception $\gamma=\left\lfloor\frac{k+1}{2}\right\rfloor>\left\lfloor\frac{k}{2}\right\rfloor$. This can only happen if $k=2 \ell+1$ is odd, and then $\gamma=\ell+1$. For this value of $\gamma$, the list $\mathrm{I}_{k+1}$ contains only one element, viz., $B_{\ell+1, \ell+1, \ell+1}^{\#}$. By Lemma 3.1 this is generated by the three elements above the main diagonal in $\mathrm{I}_{k+1}^{\prime}$ with $k=2 \ell+1$ and $\alpha=\ell, \beta=\ell+1$.

Conversely, the diagonal elements of $\mathrm{I}_{k+1}^{\prime}$ are all listed in $\mathrm{I}_{k+1}$. The indices of any non-diagonal element form a permutation of the triple $(\beta, \beta+1, \alpha+1)$. The associated $B_{\beta, \beta+1, \alpha+1}^{\#}$ is a multiple of $B_{\beta, \beta, \alpha+1}^{\#}$ which appears together with all index permutations in $\mathrm{I}_{k+1}$ for $\gamma=\alpha+1, \delta=\beta$, except for the case $\alpha=\left\lfloor\frac{k}{2}\right\rfloor=\left\lfloor\frac{k+1}{2}\right\rfloor$. This can only happen if $k=2 \ell$ is even, and then $\alpha=\ell$ and $(\beta, \beta+1, \alpha+1)=(\ell, \ell+1, \ell+1)$. But the associated elements appear in $\mathrm{I}_{k+1}$ for $\gamma=\ell, \delta=\ell+1$.

As in Section 2. Theorem 3.2 has an immediate consequence, Theorem C, for bivariate mask symbols satisfying the higher order zero conditions. This result is of great importance for studying the properties of existing subdivision schemes and also as a starting point for the construction of new schemes.

Theorem C. A convergent bivariate subdivision scheme $\mathcal{S}_{\mathrm{a}}$ satisfies the condition $\mathrm{Z}_{k}$ if and only if its mask symbol can be written in the form

$$
\mathrm{a}(\boldsymbol{z})=\sum_{B_{\alpha, \beta, \gamma}^{\#} \in \mathrm{I}_{k}} \lambda_{\alpha, \beta, \gamma} \sigma_{\alpha, \beta, \gamma}(\boldsymbol{z}) 4 B_{\alpha, \beta, \gamma}^{\#}(\boldsymbol{z}),
$$

where $\sum \lambda_{\alpha, \beta, \gamma}=1$, and the $\sigma_{\alpha, \beta, \gamma}(\boldsymbol{z})$ are Laurent polynomials normalized by the condition $\sigma_{\alpha, \beta, \gamma}(\mathbf{1})=1$. 
Examples 3.3. We illustrate the result of Theorem 3.2 by explicitly listing the generators for small values of $k$.

$$
\begin{aligned}
& \mathrm{I}_{1}=\left\{B_{1,1,0}^{\#}, B_{1,0,1}^{\#}, B_{0,1,1}^{\#}\right\}, \\
& \mathrm{I}_{2}=\left\{B_{2,2,0}^{\#}, B_{2,0,2}^{\#}, B_{0,2,2}^{\#}, B_{1,1,1}^{\#}\right\}, \\
& \mathrm{I}_{3}=\left\{B_{3,3,0}^{\#}, B_{3,0,3}^{\#}, B_{0,3,3}^{\#}, B_{2,2,1}^{\#}, B_{2,1,2}^{\#}, B_{1,2,2}^{\#}\right\}, \\
& \mathrm{I}_{4}=\left\{B_{4,4,0}^{\#}, B_{4,0,4}^{\#}, B_{0,4,4}^{\#}, B_{3,3,1}^{\#}, B_{3,1,3}^{\#}, B_{1,3,3}^{\#}, B_{2,2,2}^{\#}\right\} .
\end{aligned}
$$

\subsection{Further properties.}

To be able to show some further properties of the box spline symbols, implying the corresponding properties of the associated subdivision schemes, we need the following auxiliary result. As usual, we write

$$
D^{(n, m)}=\frac{\partial^{n+m}}{\partial z_{1}^{n} \partial z_{2}{ }^{m}}, \quad n, m \in \mathbb{N}_{0}
$$

for mixed partial differential operators.

Lemma 3.4. The partial derivatives of the box spline symbol $B_{\alpha, \beta, \gamma}^{\#}$ are given by

$$
\begin{aligned}
\left(D^{(n, m)} B_{\alpha, \beta, \gamma}^{\#}\right)\left(z_{1}, z_{2}\right)= & \\
=\sum_{\ell=0}^{\gamma}\left(\begin{array}{c}
\gamma \\
\ell
\end{array}\right) & \left(\sum_{i=0}^{n} \frac{n !}{2^{n}}\left(\begin{array}{c}
\alpha+\ell \\
n-i
\end{array}\right)\left(\frac{1+z_{1}}{2}\right)^{\alpha+\ell-(n-i)}\left(\begin{array}{c}
\gamma-\ell \\
i
\end{array}\right)\left(\frac{z_{1}-1}{2}\right)^{\gamma-\ell-i}\right) \\
& \times\left(\sum_{j=0}^{m} \frac{m !}{2^{m}}\left(\begin{array}{c}
\beta+\ell \\
m-j
\end{array}\right)\left(\frac{1+z_{2}}{2}\right)^{\beta+\ell-(m-j)}\left(\begin{array}{c}
\gamma-\ell \\
j
\end{array}\right)\left(\frac{z_{2}-1}{2}\right)^{\gamma-\ell-j}\right) .
\end{aligned}
$$

Proof. The identity

$$
\frac{1+z_{1} z_{2}}{2}=\frac{1+z_{1}}{2} \frac{1+z_{2}}{2}+\frac{z_{1}-1}{2} \frac{z_{2}-1}{2}
$$

yields

$$
\begin{aligned}
B_{\alpha, \beta, \gamma}^{\#}\left(z_{1}, z_{2}\right) & =\left(\frac{1+z_{1}}{2}\right)^{\alpha}\left(\frac{1+z_{2}}{2}\right)^{\beta}\left(\frac{1+z_{1}}{2} \frac{1+z_{2}}{2}+\frac{z_{1}-1}{2} \frac{z_{2}-1}{2}\right)^{\gamma} \\
& =\sum_{\ell=0}^{\gamma}\left(\begin{array}{l}
\gamma \\
\ell
\end{array}\right)\left(\frac{1+z_{1}}{2}\right)^{\alpha+\ell}\left(\frac{1+z_{2}}{2}\right)^{\beta+\ell}\left(\frac{z_{1}-1}{2}\right)^{\gamma-\ell}\left(\frac{z_{2}-1}{2}\right)^{\gamma-\ell} .
\end{aligned}
$$


From this we obtain with the Leibniz formula

$$
\begin{aligned}
& \left(D^{(n, m)} B_{\alpha, \beta, \gamma}^{\#}\right)\left(z_{1}, z_{2}\right)= \\
& =\sum_{\ell=0}^{\gamma}\left(\begin{array}{l}
\gamma \\
\ell
\end{array}\right) \frac{\partial^{n}}{\partial z_{1}^{n}}\left(\left(\frac{1+z_{1}}{2}\right)^{\alpha+\ell}\left(\frac{z_{1}-1}{2}\right)^{\gamma-\ell}\right) \frac{\partial^{m}}{\partial z_{2}^{m}}\left(\left(\frac{1+z_{2}}{2}\right)^{\beta+\ell}\left(\frac{z_{2}-1}{2}\right)^{\gamma-\ell}\right) \\
& =\sum_{\ell=0}^{\gamma}\left(\begin{array}{l}
\gamma \\
\ell
\end{array}\right)\left(\sum_{i=0}^{n}\left(\begin{array}{l}
n \\
i
\end{array}\right) \frac{(\alpha+\ell) !}{(\alpha+\ell-(n-i)) !} \frac{\left(1+z_{1}\right)^{\alpha+\ell-(n-i)}}{2^{\alpha+\ell}} \frac{(\gamma-\ell) !}{(\gamma-\ell-i) !} \frac{\left(z_{1}-1\right)^{\gamma-\ell-i}}{2^{\gamma-\ell}}\right) \\
& \times\left(\sum_{j=0}^{m}\left(\begin{array}{c}
m \\
j
\end{array}\right) \frac{(\beta+\ell) !}{(\beta+\ell-(m-j)) !} \frac{\left(1+z_{2}\right)^{\beta+\ell-(m-j)}}{2^{\beta+\ell}} \frac{(\gamma-\ell) !}{(\gamma-\ell-j) !} \frac{\left(z_{2}-1\right)^{\gamma-\ell-j}}{2^{\gamma-\ell}}\right) \\
& =\sum_{\ell=0}^{\gamma}\left(\begin{array}{l}
\gamma \\
\ell
\end{array}\right)\left(\sum_{i=0}^{n} \frac{n !}{2^{n}}\left(\begin{array}{c}
\alpha+\ell \\
n-i
\end{array}\right)\left(\frac{1+z_{1}}{2}\right)^{\alpha+\ell-(n-i)}\left(\begin{array}{c}
\gamma-\ell \\
i
\end{array}\right)\left(\frac{z_{1}-1}{2}\right)^{\gamma-\ell-i}\right) \\
& \times\left(\sum_{j=0}^{m} \frac{m !}{2^{m}}\left(\begin{array}{c}
\beta+\ell \\
m-j
\end{array}\right)\left(\frac{1+z_{2}}{2}\right)^{\beta+\ell-(m-j)}\left(\begin{array}{c}
\gamma-\ell \\
j
\end{array}\right)\left(\frac{z_{2}-1}{2}\right)^{\gamma-\ell-j}\right)
\end{aligned}
$$

as claimed.

Together with Theorem 3.2, this allows us to determine the maximal order of sum rules satisfied by a three-directional box spline symbol.

Proposition 3.5. For any triple $(\alpha, \beta, \gamma) \in \mathbb{N}_{0}^{3}$, the maximal $k$ such that $B_{\alpha, \beta, \gamma}^{\#} \in \mathcal{I}^{k}$ is given by

$$
k=\alpha+\beta+\gamma-\max \{\alpha, \beta, \gamma\} .
$$

Proof. Let $(\alpha, \beta, \gamma)=\pi\left(\alpha^{\prime}, \beta^{\prime}, \gamma^{\prime}\right)$ where $\pi$ is a permutation such that $\alpha^{\prime} \leq \beta^{\prime} \leq$ $\gamma^{\prime}$. It follows from Theorem 3.2 that

$$
B_{\alpha, \beta, \gamma}^{\#}=B_{\pi\left(\alpha^{\prime}, \beta^{\prime}, \gamma^{\prime}\right)}^{\#}=B_{\pi\left(0,0, \gamma^{\prime}-\beta^{\prime}\right)}^{\#} \cdot B_{\pi\left(\alpha^{\prime}, \beta^{\prime}, \beta^{\prime}\right)}^{\#} \in \mathcal{I}^{k}
$$

for $k=\alpha^{\prime}+\beta^{\prime}=\alpha+\beta+\gamma-\max \{\alpha, \beta, \gamma\}$.

On the other hand, applying (3.6) yields

$$
\begin{aligned}
\left(D^{(\alpha, \beta)} B_{\alpha, \beta, \gamma}^{\#}\right)(-1,-1) & =\frac{\alpha ! \beta !}{2^{\alpha+\beta}} \neq 0, \\
\left(D^{(\alpha, \gamma)} B_{\alpha, \beta, \gamma}^{\#}\right)(-1,1) & =(-1)^{\gamma} \frac{\alpha ! \gamma !}{2^{\alpha+\gamma}} \neq 0, \\
\text { and } \quad\left(D^{(\gamma, \beta)} B_{\alpha, \beta, \gamma}^{\#}\right)(1,-1) & =(-1)^{\gamma} \frac{\beta ! \gamma !}{2^{\beta+\gamma}} \neq 0 .
\end{aligned}
$$

This shows that $B_{\alpha, \beta, \gamma}^{\#} \notin \mathcal{I}^{k+1}$ for

$$
k=\min \{\alpha+\beta, \alpha+\gamma, \beta+\gamma\}=\alpha+\beta+\gamma-\max \{\alpha, \beta, \gamma\},
$$

and this completes the proof. 
The sets of generators described in Theorem 3.2 are minimal generating sets, as the following shows.

Proposition 3.6. The set $\mathrm{I}_{k}$ generating $\mathcal{I}^{k}$ is minimal in the sense that for any $B_{\alpha, \beta, \gamma}^{\#} \in \mathrm{I}_{k}$, the reduced set $\mathrm{I}_{k} \backslash\left\{B_{\alpha, \beta, \gamma}^{\#}\right\}$ does no longer generate the ideal $\mathcal{I}^{k}$.

Proof. We begin with an element of the form $B_{\alpha, \beta, \beta}^{\#} \in \mathrm{I}_{k}$. We find that

$$
\left(D^{(\alpha, \beta)} B_{\alpha, \beta, \beta}^{\#}\right)(-1,-1)=\frac{\alpha ! \beta !}{2^{\alpha+\beta}} \neq 0,
$$

and we claim that for all other elements $B_{\widetilde{\alpha}, \widetilde{\beta}, \widetilde{\gamma}}^{\#} \in \widetilde{\mathrm{I}}_{k}:=\mathrm{I}_{k} \backslash\left\{B_{\alpha, \beta, \beta}^{\#}\right\}$, we have

$$
\left(D^{(n, m)} B_{\widetilde{\alpha}, \widetilde{\beta}, \widetilde{\gamma}}^{\#}\right)(-1,-1)=0 \text { for all }(0,0) \leq(n, m) \leq(\alpha, \beta) .
$$

To this end, assume first that $\alpha<\beta$, then we have

$$
\left(D^{(n, m)} B_{\beta, \alpha, \beta}^{\#}\right)(-1,-1)=0 \quad \text { and } \quad\left(D^{(n, m)} B_{\beta, \beta, \alpha}^{\#}\right)(-1,-1)=0,
$$

since in (3.6), we have $\beta+\ell-(n-i) \geq \beta-\alpha>0$ and therefore we also have $\left(\frac{1+z_{1}}{2}\right)^{\beta+\ell-(n-i)}=0$ for $z_{1}=-1$. For arbitrary $\alpha \leq \beta$, consider $\left(\alpha^{\prime}, \beta^{\prime}\right) \neq$ $(\alpha, \beta)$.

In case $\alpha^{\prime}<\alpha$ and thus $\beta^{\prime}>\beta$, we have

$$
\left(D^{(n, m)} B_{\alpha^{\prime}, \beta^{\prime}, \beta^{\prime}}^{\#}\right)(-1,-1)=0
$$

since in (3.6), we have $\beta^{\prime}+\ell-(m-j) \geq \beta^{\prime}-\beta>0$ and therefore we get $\left(\frac{1+z_{2}}{2}\right)^{\beta^{\prime}+\ell-(m-j)}=0$ for $z_{2}=-1$; and also

$$
\left(D^{(n, m)} B_{\beta^{\prime}, \alpha^{\prime}, \beta^{\prime}}^{\#}\right)(-1,-1)=0 \quad \text { and } \quad\left(D^{(n, m)} B_{\beta^{\prime}, \beta^{\prime}, \alpha^{\prime}}^{\#}\right)(-1,-1)=0
$$

since in (3.6), we have $\beta^{\prime}+\ell-(n-i) \geq \beta^{\prime}-\alpha>0$ and therefore we also get $\left(\frac{1+z_{1}}{2}\right)^{\beta^{\prime}+\ell-(n-i)}=0$ for $z_{1}=-1$.

In case $\alpha<\alpha^{\prime} \leq \beta^{\prime}<\beta$, (3.9) holds since in (3.6), we have $\alpha^{\prime}+\ell-(n-i) \geq$ $\alpha^{\prime}-\alpha>0$ and therefore $\left(\frac{1+z_{1}}{2}\right)^{\alpha^{\prime}+\ell-(n-i)}=0$ for $z_{1}=-1$; and also (3.10) holds since in (3.6), we have $\beta^{\prime}+\ell-(n-i) \geq \beta^{\prime}-\alpha>0$ and therefore $\left(\frac{1+z_{1}}{2}\right)^{\beta^{\prime}+\ell-(n-i)}=0$ for $z_{1}=-1$. So (3.8) is shown.

But this implies that

$$
B_{\alpha, \beta, \beta}^{\#} \notin<\widetilde{\mathrm{I}}_{k}>
$$

since otherwise, we could write

$$
B_{\alpha, \beta, \beta}^{\#}=\sum_{B_{\widetilde{\alpha}, \widetilde{\beta}, \widetilde{\gamma}}^{\#} \in \widetilde{\mathrm{I}}_{k}} p_{\widetilde{\alpha}, \widetilde{\beta}, \widetilde{\gamma}} B_{\widetilde{\alpha}, \widetilde{\beta}, \widetilde{\gamma}}^{\#}
$$


which, employing the Leibniz formula, yields

$$
\begin{aligned}
&\left(D^{(\alpha, \beta)} B_{\alpha, \beta, \beta}^{\#}\right)(-1,-1)= \\
&=\sum_{B_{\tilde{\alpha}, \tilde{\beta}, \tilde{\gamma}}^{\#} \in \widetilde{I}_{k}} \sum_{n=0}^{\alpha}\left(\begin{array}{l}
\alpha \\
n
\end{array}\right) \sum_{m=0}^{\beta}\left(\begin{array}{c}
\beta \\
m
\end{array}\right)\left(D^{(\alpha-n, \beta-m)} p_{\widetilde{\alpha}, \widetilde{\beta}, \widetilde{\gamma}}\right)(-1,-1) \\
& \quad \times\left(D^{(n, m)} B_{\widetilde{\alpha}, \widetilde{\beta}, \widetilde{\gamma}}^{\#}\right)(-1,-1),
\end{aligned}
$$

and this contradicts (3.7) and (3.8).

By symmetry in $z_{1}$ and $z_{2}$, it follows that also

$$
B_{\beta, \alpha, \beta}^{\#} \notin<\mathrm{I}_{k} \backslash\left\{B_{\beta, \alpha, \beta}^{\#}\right\}>.
$$

It remains to show that

$$
B_{\beta, \beta, \alpha}^{\#} \notin<\mathrm{I}_{k} \backslash\left\{B_{\beta, \beta, \alpha}^{\#}\right\}>.
$$

This can be achieved by employing a directional derivative and considering mixed derivatives of the form

$$
D^{(n, m, \ell)}=\left(\frac{\partial}{\partial z_{1}}\right)^{n}\left(\frac{\partial}{\partial z_{2}}\right)^{m}\left(\frac{\partial}{\partial z_{1}}+\frac{\partial}{\partial z_{2}}\right)^{\ell}=\sum_{j=0}^{\ell}\left(\begin{array}{l}
\ell \\
j
\end{array}\right) D^{(n+j, m+\ell-j)} .
$$

Along the same lines as above, one shows that

$$
\left(D^{(\alpha, 0, \beta)} B_{\beta, \beta, \alpha}^{\#}\right)(1,-1)=(-1)^{\alpha} \frac{\alpha ! \beta !}{2^{\alpha+\beta}} \neq 0,
$$

but that for all other elements $B_{\widetilde{\alpha}, \widetilde{\beta}, \widetilde{\gamma}}^{\#} \in \mathrm{I}_{k} \backslash\left\{B_{\beta, \beta, \alpha}^{\#}\right\}$,

$$
\left(D^{(n, 0, \ell)} B_{\widetilde{\alpha}, \widetilde{\beta}, \widetilde{\gamma}}^{\#}\right)(1,-1)=0 \quad \text { for all }(0,0) \leq(n, \ell) \leq(\alpha, \beta) .
$$

Alternatively, we may employ the coordinate transformation

$$
\left(\begin{array}{l}
w_{1} \\
w_{2}
\end{array}\right)=\left(\begin{array}{c}
z_{1} z_{2} \\
1 / z_{2}
\end{array}\right) \quad \Longleftrightarrow \quad\left(\begin{array}{c}
z_{1} \\
z_{2}
\end{array}\right)=\left(\begin{array}{c}
w_{1} w_{2} \\
1 / w_{2}
\end{array}\right)
$$

which yields

$$
B_{\alpha, \beta, \gamma}^{\#}\left(z_{1}, z_{2}\right)=\frac{1}{w_{2}^{\beta}} B_{\gamma, \beta, \alpha}^{\#}\left(w_{1}, w_{2}\right)
$$

and thus allows us to reduce this case to the first one also.

The characterization of convergent bivariate subdivision schemes established in Theorem $\mathrm{C}$ opens a way for their systematic study. We only point out the following facts: 
- The set $\mathrm{I}_{k}$ of generators for $\mathcal{I}^{k}$ is symmetric in the sense that it is invariant under an interchange of the two variables, and that the indices of the generators can be permuted arbitrarily.

- For even $k$, the $k / 2$-th power of (the symbol of) the Courant hat function $B_{1,1,1}$ appears in $\mathrm{I}_{k}$.

- Most interesting for us, however, is the fact that the smoothness of these generators matches perfectly with the order of polynomial reproduction. More generally, the box spline symbol $B_{\alpha, \beta, \gamma}^{\#}$ is an element of

$$
L_{\infty}^{(\kappa-1)} \subset C^{(\kappa-2)} \quad \text { for } \quad \kappa=\alpha+\beta+\gamma-\max \{\alpha, \beta, \gamma\}=k,
$$

see 2]. This means that for each three-directional box spline, smoothness and polynomial reproduction match in the same way as in the univariate case. This property is not necessarily preserved under taking combinations as described in Theorem $\mathrm{C}$, however, as we shall see below for the fourdirectional box splines.

- It may also be of interest that the total degree of the box spline with the symbol $B_{\alpha, \beta, \gamma}^{\#}$ is

\[ \mu=\alpha+\beta+\gamma-2, \]
which for the generators $B_{\beta, \beta, \alpha}^{\#}, B_{\beta, \alpha, \beta}^{\#}$, and $B_{\alpha, \beta, \beta}^{\#}$ with $\alpha \leq \beta$ yields

$$
\mu=\alpha+2 \beta-2=2 k-\alpha-2=2 \kappa-\alpha-2 .
$$

\subsection{Modification.}

As in Section 2, we can replace the $q_{\Theta}$ by the $\widetilde{q}_{\Theta}$, and all results still hold. More precisely, this means replacing $B_{\alpha, \beta, \gamma}^{\#}$ by

$$
\widetilde{B}_{\alpha, \beta, \gamma}^{\#}\left(z_{1}, z_{2}\right)=\left(\frac{1+z_{1}}{2}\right)^{\alpha}\left(\frac{1+z_{2}}{2}\right)^{\beta}\left(\frac{z_{1}+z_{2}}{2}\right)^{\gamma} .
$$

For example, Lemma 3.1 holds as stated, and in its proof, we only have to replace (3.4) by

$$
\widetilde{B}_{1,0,0}^{\#}\left(z_{1}, z_{2}\right)+\widetilde{B}_{0,1,0}^{\#}\left(z_{1}, z_{2}\right)-\widetilde{B}_{0,0,1}^{\#}\left(z_{1}, z_{2}\right)=1 .
$$

Also, Theorem 3.2 together with its proof, Examples 3.3 and Theorem $\mathrm{C}$ are still valid. Lemma 3.4 now runs as follows.

Lemma 3.7. The partial derivatives of the modified box spline symbol $\widetilde{B}_{\alpha, \beta, \gamma}^{\#}$ are given by

$$
\begin{aligned}
\left(D^{(n, m)} \widetilde{B}_{\alpha, \beta, \gamma}^{\#}\right)\left(z_{1}, z_{2}\right)= & \\
=\sum_{\ell=0}^{\gamma}\left(\begin{array}{c}
\gamma \\
\ell
\end{array}\right) & \left(\sum_{i=0}^{n} \frac{n !}{2^{n}}\left(\begin{array}{c}
\alpha \\
n-i
\end{array}\right)\left(\frac{1+z_{1}}{2}\right)^{\alpha-(n-i)}\left(\begin{array}{l}
\ell \\
i
\end{array}\right)\left(\frac{z_{1}}{2}\right)^{\ell-i}\right) \\
& \times\left(\sum_{j=0}^{m} \frac{m !}{2^{m}}\left(\begin{array}{c}
\beta \\
m-j
\end{array}\right)\left(\frac{1+z_{2}}{2}\right)^{\beta-(m-j)}\left(\begin{array}{c}
\gamma-\ell \\
j
\end{array}\right)\left(\frac{z_{2}}{2}\right)^{\gamma-\ell-j}\right) .
\end{aligned}
$$


Proof. Writing

$$
\begin{aligned}
\widetilde{B}_{\alpha, \beta, \gamma}^{\#}\left(z_{1}, z_{2}\right) & =\left(\frac{1+z_{1}}{2}\right)^{\alpha}\left(\frac{1+z_{2}}{2}\right)^{\beta}\left(\frac{z_{1}}{2}+\frac{z_{2}}{2}\right)^{\gamma} \\
& =\sum_{\ell=0}^{\gamma}\left(\begin{array}{l}
\gamma \\
\ell
\end{array}\right)\left(\frac{1+z_{1}}{2}\right)^{\alpha}\left(\frac{1+z_{2}}{2}\right)^{\beta}\left(\frac{z_{1}}{2}\right)^{\ell}\left(\frac{z_{2}}{2}\right)^{\gamma-\ell}
\end{aligned}
$$

yields by the Leibniz formula

$$
\begin{aligned}
& \left(D^{(n, m)} \widetilde{B}_{\alpha, \beta, \gamma}^{\#}\right)\left(z_{1}, z_{2}\right)= \\
& =\sum_{\ell=0}^{\gamma}\left(\begin{array}{l}
\gamma \\
\ell
\end{array}\right) \frac{\partial^{n}}{\partial z_{1}{ }^{n}}\left(\left(\frac{1+z_{1}}{2}\right)^{\alpha}\left(\frac{z_{1}}{2}\right)^{\ell}\right) \frac{\partial^{m}}{\partial z_{2}{ }^{m}}\left(\left(\frac{1+z_{2}}{2}\right)^{\beta}\left(\frac{z_{2}}{2}\right)^{\gamma-\ell}\right) \\
& =\sum_{\ell=0}^{\gamma}\left(\begin{array}{l}
\gamma \\
\ell
\end{array}\right)\left(\sum_{i=0}^{n}\left(\begin{array}{l}
n \\
i
\end{array}\right) \frac{\alpha !}{(\alpha-(n-i)) !} \frac{\left(1+z_{1}\right)^{\alpha-(n-i)}}{2^{\alpha}} \frac{\ell !}{(\ell-i) !} \frac{z_{1}^{\ell-i}}{2^{\ell}}\right) \\
& \times\left(\sum_{j=0}^{m}\left(\begin{array}{c}
m \\
j
\end{array}\right) \frac{\beta !}{(\beta-(m-j)) !} \frac{\left(1+z_{2}\right)^{\beta-(m-j)}}{2^{\beta}} \frac{(\gamma-\ell) !}{(\gamma-\ell-j) !} \frac{z_{2}^{\gamma-\ell-j}}{2^{\gamma-\ell}}\right) \\
& =\sum_{\ell=0}^{\gamma}\left(\begin{array}{l}
\gamma \\
\ell
\end{array}\right)\left(\sum_{i=0}^{n} \frac{n !}{2^{n}}\left(\begin{array}{c}
\alpha \\
n-i
\end{array}\right)\left(\frac{1+z_{1}}{2}\right)^{\alpha-(n-i)}\left(\begin{array}{l}
\ell \\
i
\end{array}\right)\left(\frac{z_{1}}{2}\right)^{\ell-i}\right) \\
& \times\left(\sum_{j=0}^{m} \frac{m !}{2^{m}}\left(\begin{array}{c}
\beta \\
m-j
\end{array}\right)\left(\frac{1+z_{2}}{2}\right)^{\beta-(m-j)}\left(\begin{array}{c}
\gamma-\ell \\
j
\end{array}\right)\left(\frac{z_{2}}{2}\right)^{\gamma-\ell-j}\right)
\end{aligned}
$$

as claimed.

This implies that Proposition 3.5 remains valid, and in its proof, only the values of the listed nonzero mixed partial derivatives need to be multiplied by $(-1)^{\gamma}$. Also, Proposition 3.6 still holds as stated: in its proof, once more the signs of the nonzero derivative values change, and in the last part, we need to consider the directional derivative $\frac{\partial}{\partial z_{1}}-\frac{\partial}{\partial z_{2}}$ instead of $\frac{\partial}{\partial z_{1}}+\frac{\partial}{\partial z_{2}}$; alternatively, we can employ the coordinate transformation

$$
\left(\begin{array}{c}
w_{1} \\
w_{2}
\end{array}\right)=\left(\begin{array}{c}
z_{1} / z_{2} \\
1 / z_{2}
\end{array}\right) \quad \Longleftrightarrow \quad\left(\begin{array}{c}
z_{1} \\
z_{2}
\end{array}\right)=\left(\begin{array}{c}
w_{1} / w_{2} \\
1 / w_{2}
\end{array}\right)
$$

which yields

$$
\widetilde{B}_{\alpha, \beta, \gamma}^{\#}\left(z_{1}, z_{2}\right)=\frac{1}{w_{2}^{\alpha+\beta+\gamma}} \widetilde{B}_{\gamma, \beta, \alpha}^{\#}\left(w_{1}, w_{2}\right)
$$

Another approach is the use of the coordinate transformation

$$
\left(\begin{array}{c}
w_{1} \\
w_{2}
\end{array}\right)=\left(\begin{array}{c}
z_{1} \\
1 / z_{2}
\end{array}\right) \quad \Longleftrightarrow \quad\left(\begin{array}{c}
z_{1} \\
z_{2}
\end{array}\right)=\left(\begin{array}{c}
w_{1} \\
1 / w_{2}
\end{array}\right)
$$


which was already mentioned at the beginning of Section 2 This yields

$$
B_{\alpha, \beta, \gamma}^{\#}\left(z_{1}, z_{2}\right)=\frac{1}{w_{2}^{\beta+\gamma}} \widetilde{B}_{\alpha, \beta, \gamma}^{\#}\left(w_{1}, w_{2}\right)
$$

which shows that all the results for the $B_{\alpha, \beta, \gamma}^{\#}$ hold for the $\widetilde{B}_{\alpha, \beta, \gamma}^{\#}$ also.

\subsection{Four-directional box splines}

The refinement mask symbol of a four-directional box spline has the form

$$
4 B_{\alpha, \beta, \gamma, \delta}^{\#}\left(z_{1}, z_{2}\right)=4\left(\frac{1+z_{1}}{2}\right)^{\alpha}\left(\frac{1+z_{2}}{2}\right)^{\beta}\left(\frac{1+z_{1} z_{2}}{2}\right)^{\gamma}\left(\frac{1+z_{1} / z_{2}}{2}\right)^{\delta} .
$$

This uses both alternatives, $\boldsymbol{e}_{1}+\boldsymbol{e}_{2}$ and $\boldsymbol{e}_{1}-\boldsymbol{e}_{2}$, for the third direction discussed above. We can rewrite the above as

$$
B_{\alpha, \beta, \gamma, \delta}^{\#}\left(z_{1}, z_{2}\right)=\frac{1}{2^{\delta}}\left(1+\frac{z_{1}}{z_{2}}\right)^{\delta} B_{\alpha, \beta, \gamma}^{\#}\left(z_{1}, z_{2}\right),
$$

illustrating the well-known fact that any four-directional box spline is indeed a special convex combination of the shifts of some three-directional box spline. This convex combination uses the normalized binomial weights $\lambda_{\delta, \ell}=2^{-\delta}\left(\begin{array}{l}\delta \\ \ell\end{array}\right)$, $\ell=0, \ldots, \delta$. The representation in (3.12) is not optimal, however, if one tries to determine the maximal $k$ such that $B_{\alpha, \beta, \gamma, \delta}^{\#} \in \mathcal{I}^{k}$. Instead, we use the identity $z_{1}+z_{2}=\left(1+z_{1}\right)\left(1+z_{2}\right)-\left(1+z_{1} z_{2}\right)$ which in terms of the normalized box spline symbols reads as

$$
B_{0,0,0,1}^{\#}\left(z_{1}, z_{2}\right)=\frac{1}{z_{2}} \frac{z_{1}+z_{2}}{2}=\frac{1}{z_{2}}\left(2 B_{1,1,0}^{\#}\left(z_{1}, z_{2}\right)-B_{0,0,1}^{\#}\left(z_{1}, z_{2}\right)\right) .
$$

This yields

$$
\begin{aligned}
B_{\alpha, \beta, \gamma, \delta}^{\#}\left(z_{1}, z_{2}\right) & =B_{\alpha, \beta, \gamma}^{\#}\left(z_{1}, z_{2}\right) \frac{1}{z_{2}^{\delta}}\left(2 B_{1,1,0}^{\#}\left(z_{1}, z_{2}\right)-B_{0,0,1}^{\#}\left(z_{1}, z_{2}\right)\right)^{\delta} \\
& =\sum_{\ell=0}^{\delta} \frac{2^{\ell}(-1)^{\delta-\ell}}{z_{2}^{\delta}}\left(\begin{array}{l}
\delta \\
\ell
\end{array}\right) B_{\alpha+\ell, \beta+\ell, \gamma+\delta-\ell}^{\#}\left(z_{1}, z_{2}\right) .
\end{aligned}
$$

Proposition 3.8. For any quadruple $(\alpha, \beta, \gamma, \delta) \in \mathbb{N}_{0}^{4}$, the maximal $k$ such that $B_{\alpha, \beta, \gamma, \delta}^{\#} \in \mathcal{I}^{k}$ is given by

$$
k=\alpha+\beta+\gamma+\delta-\max \{\alpha, \beta, \gamma+\delta\} .
$$

In other words, $B_{\alpha, \beta, \gamma, \delta}^{\#} \in \mathcal{I}^{k}$ if and only if $B_{\alpha, \beta, \gamma+\delta}^{\#} \in \mathcal{I}^{k}$.

Proof. By Proposition 3.5. we may conclude from (3.13) that $B_{\alpha, \beta, \gamma, \delta}^{\#} \in \mathcal{I}^{k}$ for

$$
\begin{aligned}
k & =\min _{\ell=0, \ldots, \delta}\{\alpha+\beta+\gamma+\delta+\ell-\max \{\alpha+\ell, \beta+\ell, \gamma+\delta-\ell\}\} \\
& =\min _{\ell=0, \ldots, \delta}\{\min \{\beta+\gamma+\delta, \alpha+\gamma+\delta, \alpha+\beta+2 \ell\}\} \\
& =\min \{\beta+\gamma+\delta, \alpha+\gamma+\delta, \alpha+\beta\} \\
& =\alpha+\beta+\gamma+\delta-\max \{\alpha, \beta, \gamma+\delta\} .
\end{aligned}
$$


Thus it remains to show that $B_{\alpha, \beta, \gamma, \delta}^{\#} \notin \mathcal{I}^{k+1}$ for this $k$. Equivalently, we show that

$\widetilde{B}_{\alpha, \beta, \gamma, \delta}^{\#}\left(z_{1}, z_{2}\right)=z_{2}^{\delta} B_{\alpha, \beta, \gamma, \delta}^{\#}\left(z_{1}, z_{2}\right)=\left(\frac{1+z_{1}}{2}\right)^{\alpha}\left(\frac{1+z_{2}}{2}\right)^{\beta}\left(\frac{1+z_{1} z_{2}}{2}\right)^{\gamma}\left(\frac{z_{1}+z_{2}}{2}\right)^{\delta}$

satisfies $\widetilde{B}_{\alpha, \beta, \gamma, \delta}^{\#} \notin \mathcal{I}^{k+1}$ for this $k$.

To this end, we proceed along the lines of the proofs of Lemmata 3.4 and 3.7 and Proposition 3.5. Writing

$$
\begin{gathered}
\widetilde{B}_{\alpha, \beta, \gamma, \delta}^{\#}\left(z_{1}, z_{2}\right)=\left(\frac{1+z_{1}}{2}\right)^{\alpha}\left(\frac{1+z_{2}}{2}\right)^{\beta}\left(\frac{1+z_{1}}{2} \frac{1+z_{2}}{2}+\frac{z_{1}-1}{2} \frac{z_{2}-1}{2}\right)^{\gamma}\left(\frac{z_{1}}{2}+\frac{z_{2}}{2}\right)^{\delta} \\
=\sum_{\ell=0}^{\gamma}\left(\begin{array}{l}
\gamma \\
\ell
\end{array}\right) \sum_{j=0}^{\delta}\left(\begin{array}{l}
\delta \\
j
\end{array}\right)\left(\frac{1+z_{1}}{2}\right)^{\alpha+\ell}\left(\frac{z_{1}-1}{2}\right)^{\gamma-\ell}\left(\frac{z_{1}}{2}\right)^{j} \\
\times\left(\frac{1+z_{2}}{2}\right)^{\beta+\ell}\left(\frac{z_{2}-1}{2}\right)^{\gamma-\ell}\left(\frac{z_{2}}{2}\right)^{\delta-j}
\end{gathered}
$$

yields

$$
\begin{aligned}
& \left(D^{(n, m)} \widetilde{B}_{\alpha, \beta, \gamma, \delta}^{\#}\right)\left(z_{1}, z_{2}\right)=\sum_{\ell=0}^{\gamma}\left(\begin{array}{c}
\gamma \\
\ell
\end{array}\right) \\
& \quad \times \sum_{j=0}^{\delta}\left(\begin{array}{c}
\delta \\
j
\end{array}\right)\left(\sum_{\sum n_{i}=n} \frac{n !}{2^{n}}\left(\begin{array}{c}
\alpha+\ell \\
n_{1}
\end{array}\right)\left(\frac{1+z_{1}}{2}\right)^{\alpha+\ell-n_{1}}\left(\begin{array}{c}
\gamma-\ell \\
n_{2}
\end{array}\right)\left(\frac{z_{1}-1}{2}\right)^{\gamma-\ell-n_{2}}\left(\begin{array}{c}
j \\
n_{3}
\end{array}\right)\left(\frac{z_{1}}{2}\right)^{j-n_{3}}\right) \\
& \quad \times\left(\sum_{\sum m_{i}=m} \frac{m !}{2^{m}}\left(\begin{array}{c}
\beta+\ell \\
m_{1}
\end{array}\right)\left(\frac{1+z_{2}}{2}\right)^{\beta+\ell-m_{1}}\left(\begin{array}{c}
\gamma-\ell \\
m_{2}
\end{array}\right)\left(\frac{z_{2}-1}{2}\right)^{\gamma-\ell-m_{2}}\left(\begin{array}{c}
\delta-j \\
m_{3}
\end{array}\right)\left(\frac{z_{2}}{2}\right)^{\delta-j-m_{3}}\right)
\end{aligned}
$$

and, therefore,

$$
\begin{aligned}
&\left(D^{(\alpha, \beta)} \widetilde{B}_{\alpha, \beta, \gamma, \delta}^{\#}\right)(-1,-1)=(-1)^{\delta} \frac{\alpha ! \beta !}{2^{\alpha+\beta}} \neq 0, \\
&\left(D^{(\alpha, \gamma+\delta)} \widetilde{B}_{\alpha, \beta, \gamma, \delta}^{\#}\right)(-1,1)=(-1)^{\gamma} \frac{\alpha !(\gamma+\delta) !}{2^{\alpha+\gamma+\delta}} \neq 0, \\
& \text { and } \quad\left(D^{(\gamma+\delta, \beta)} \widetilde{B}_{\alpha, \beta, \gamma, \delta}^{\#}\right)(1,-1)=(-1)^{\gamma} \frac{\beta !(\gamma+\delta) !}{2^{\beta+\gamma+\delta}} \neq 0 .
\end{aligned}
$$

This shows that $\widetilde{B}_{\alpha, \beta, \gamma, \delta}^{\#} \notin \mathcal{I}^{k+1}$ for

$$
k=\min \{\alpha+\beta, \alpha+\gamma+\delta, \beta+\gamma+\delta\}=\alpha+\beta+\gamma+\delta-\max \{\alpha, \beta, \gamma+\delta\},
$$

and this completes the proof.

Remark. The proof of Proposition 3.8 establishes the connection between the order $k$ in (3.14) and the smoothness of the $(\alpha, \beta, \gamma, \delta)$-box spline, which can 
be determined using the results in [1]. The function is an element of $L_{\infty}^{(\kappa-1)} \subset$ $C^{(\kappa-2)}$ for

$\kappa=\min \{\alpha+\beta+\gamma, \alpha+\beta+\delta, \alpha+\gamma+\delta, \beta+\gamma+\delta\}=\alpha+\beta+\gamma+\delta-\max \{\alpha, \beta, \gamma, \delta\}$, while the order $k$ (degree $k-1$ ) of polynomial reproduction is given by

$$
k=\min \{\alpha+\beta, \alpha+\gamma+\delta, \beta+\gamma+\delta\}=\alpha+\beta+\gamma+\delta-\max \{\alpha, \beta, \gamma+\delta\} .
$$

Corollary 3.9. For the four-directional $(\alpha, \beta, \gamma, \delta)$-box spline, we have $\kappa \geq k$, and

$$
\kappa>k \Longleftrightarrow \gamma+\delta>\max \{\alpha, \beta\} \quad \text { and } \min \{\gamma, \delta\}>0
$$

Proof. By the above remark,

$$
\kappa-k=\max \{\alpha, \beta, \gamma+\delta\}-\max \{\alpha, \beta, \gamma, \delta\} \geq 0 .
$$

In the case $\max \{\alpha, \beta, \gamma+\delta\}=\max \{\alpha, \beta\}$, we have $\max \{\gamma, \delta\} \leq \gamma+\delta \leq \max \{\alpha, \beta\}$, i. e., also $\max \{\alpha, \beta, \gamma, \delta\}=\max \{\alpha, \beta\}$ and therefore $\kappa=k$. Otherwise, we have $\gamma+\delta>\max \{\alpha, \beta\}$, and then

$$
\kappa-k=\gamma+\delta-\max \{\alpha, \beta, \gamma, \delta\}=\min \{\gamma+\delta-\alpha, \gamma+\delta-\beta, \gamma, \delta\}
$$

where the first two elements are positive, so in this case,

$$
\kappa-k=0 \quad \Longleftrightarrow \quad \min \{\gamma, \delta\}=0 .
$$

All in all, this yields that

$$
\kappa-k=0 \quad \Longleftrightarrow \quad \gamma+\delta \leq \max \{\alpha, \beta\} \quad \text { or } \quad \min \{\gamma, \delta\}=0,
$$

which proves the claim.

The condition $\min \{\gamma, \delta\}=0$ is worth a closer look. For $\delta=0$, we are in the standard three-directional case, while for $\gamma=0$, we have

$$
B_{\alpha, \beta, 0, \delta}^{\#}\left(z_{1}, z_{2}\right)=z_{2}^{\beta} B_{\alpha, \beta, \delta}^{\#}\left(z_{1}, 1 / z_{2}\right)=z_{2}^{-\delta} \widetilde{B}_{\alpha, \beta, \delta}^{\#}\left(z_{1}, z_{2}\right),
$$

so these are three-directional splines on the reflected grid.

Examples 3.10. We list a few standard examples together with the decompositions of their mask symbols according to (3.13) and to (3.5).

The (1,1,1,1)-box spline, known as the Zwart-Powell element, has the mask symbol

$$
\begin{aligned}
4 B_{1,1,1,1}^{\#}\left(z_{1}, z_{2}\right) & =\frac{4}{z_{2}}\left(2 B_{2,2,1}^{\#}\left(z_{1}, z_{2}\right)-B_{1,1,2}^{\#}\left(z_{1}, z_{2}\right)\right) \\
& =1 \cdot \frac{1+z_{1} / z_{2}}{2} \cdot 4 B_{1,1,1}^{\#}\left(z_{1}, z_{2}\right) .
\end{aligned}
$$


We find $k=4-2=2$, so the associated subdivision scheme reproduces polynomials of total degree up to one; but $\kappa=4-1=3$, so the function is in $L_{\infty}^{(2)} \subset C^{(1)}$.

The $(2,2,1,1)$-box spline has the mask symbol

$$
\begin{aligned}
4 B_{2,2,1,1}^{\#}\left(z_{1}, z_{2}\right) & =\frac{4}{z_{2}}\left(2 B_{3,3,1}^{\#}\left(z_{1}, z_{2}\right)-B_{2,2,2}^{\#}\left(z_{1}, z_{2}\right)\right) \\
& =2 \cdot \frac{1}{z_{2}} \cdot 4 B_{3,3,1}^{\#}\left(z_{1}, z_{2}\right)+(-1) \cdot \frac{1}{z_{2}} \cdot 4 B_{2,2,2}^{\#}\left(z_{1}, z_{2}\right) .
\end{aligned}
$$

Here, we have $k=6-2=4$, telling us that polynomials of degree up to 3 are reproduced, and also $\kappa=6-2=4$, i. e., the function is an element of $L_{\infty}^{(3)} \subset C^{(2)}$.

More interesting are the higher order four-directional splines. For example, the $(4,4,1,1)$-box spline has order of polynomial reproduction $k=10-4=6$ and $\kappa=10-4=6$. Its mask symbol can be represented as

$$
\begin{aligned}
4 B_{4,4,1,1}^{\#}\left(z_{1}, z_{2}\right) & =\frac{4}{z_{2}}\left(2 B_{5,5,1}^{\#}-B_{4,4,2}^{\#}\left(z_{1}, z_{2}\right)\right) \\
& =2 \cdot \frac{1}{z_{2}} \cdot 4 B_{5,5,1}^{\#}\left(z_{1}, z_{2}\right)+(-1) \cdot \frac{1}{z_{2}} \cdot 4 B_{4,4,2}^{\#}\left(z_{1}, z_{2}\right) .
\end{aligned}
$$

Remark 3.11. The vector case, i. e., the case when the matrix mask $\mathrm{A} \in \ell_{0}^{s \times s}\left(\mathbb{Z}^{d}\right)$ is a finitely supported sequence of $s \times s$-matrices indexed by $\mathbb{Z}^{d}$, is more intricate. The formulation of the zero conditions (1.7) for the mask symbol of multivariate vector subdivision schemes depends greatly on the so-called rank of the scheme, see [6, 12, 26]. Such a formulation, see, e.g., 22], does not allow us to read off the properties of the entries of the matrix Laurent polynomial $\mathrm{A}(\boldsymbol{z})$ directly. It is possible, though, to use a slight modification of the transformation in 22] to obtain the matrix sequences $\mathrm{T}, \mathrm{T}^{i n v} \in \ell_{0}^{s \times s}\left(\mathbb{Z}^{d}\right)$ such that

$$
\widetilde{\mathrm{A}}(\boldsymbol{z})=\mathrm{T}^{i n v}\left(\boldsymbol{z}^{2}\right) \cdot \mathrm{A}(\boldsymbol{z}) \cdot \mathrm{T}(\boldsymbol{z})
$$

satisfies the zero conditions of a form that makes the structure of some of the entries of $\mathrm{A}(\boldsymbol{z})$ more evident. However, this is a topic for further investigations.

\section{Examples}

In this section we illustrate the result of Theorem $\mathrm{C}$ with some examples. We would like to emphasize that this result does not only simplify the study of the properties of subdivision schemes, but also yields a way for enhancing certain properties of existing schemes by combining them appropriately.

In the following, the set $\mathrm{I}_{k}$ of the box spline symbols is the set of generators for $\mathcal{I}^{k}$ as in Theorem 3.2

In the masks displayed below the boldface entry at bottom-left position refers to the index $(0,0)$. This assumption is not really important, but as stated above already, we prefer to shift masks so that they are supported in the positive quadrant and have polynomial symbols. 


\subsection{A bivariate interpolatory scheme}

Interpolatory schemes are characterized by the fact that one of the submasks is a $\delta$ sequence, or equivalently, one of the subsymbols is identically one. Theorem $\mathrm{C}$ allows us to present a systematic way for creating interpolatory schemes from our lists of generators by equating the coefficients of their affine combinations and normalizing them appropriately.

To provide just one such example, consider the interpolatory scheme studied in 23, Example 2], a bivariate version of the univariate four-point interpolation scheme given in [18]. Its mask is

$$
\mathrm{a}=\frac{1}{32}\left(\begin{array}{ccccccc}
0 & 0 & -1 & -2 & -1 & 0 & 0 \\
0 & 0 & 0 & 0 & 0 & 0 & 0 \\
-1 & 0 & 10 & 18 & 10 & 0 & -1 \\
-2 & 0 & 18 & 32 & 18 & 0 & -2 \\
-1 & 0 & 10 & 18 & 10 & 0 & -1 \\
0 & 0 & 0 & 0 & 0 & 0 & 0 \\
0 & 0 & -1 & -2 & -1 & 0 & 0
\end{array}\right) .
$$

The scheme reproduces polynomials up to degree $k-1=3$, whence a $(\boldsymbol{z}) \in \mathcal{I}^{4}$, and a representation of $\mathrm{a}(\boldsymbol{z})$ in terms of three-directional box splines from the list $\mathrm{I}_{4}$ is given by

$$
\begin{aligned}
& z_{1}^{3} z_{2}^{3} \mathrm{a}\left(z_{1}, z_{2}\right) \\
& =-2^{4} B_{4,4,0}^{\#}\left(z_{1}, z_{2}\right)-2\left(z_{1}^{2}+z_{2}^{2}\right) B_{2,2,2}^{\#}\left(z_{1}, z_{2}\right)+2^{3}\left(1+z_{1}+z_{2}\right) B_{3,3,1}^{\#}\left(z_{1}, z_{2}\right) \\
& =4\left\{-4 B_{4,4,0}^{\#}\left(z_{1}, z_{2}\right)-\frac{z_{1}^{2}+z_{2}^{2}}{2} B_{2,2,2}^{\#}\left(z_{1}, z_{2}\right)+6 \frac{1+z_{1}+z_{2}}{3} B_{3,3,1}^{\#}\left(z_{1}, z_{2}\right)\right\} .
\end{aligned}
$$

From the second line, the weights $\lambda$ are recognized as $-4,-1$, and 6 , and the normalized $\sigma$-symbols are

$$
1, \quad \frac{z_{1}^{2}+z_{2}^{2}}{2}, \text { and } \frac{1+z_{1}+z_{2}}{3},
$$

respectively.

\subsection{The butterfly scheme}

The butterfly scheme has been studied in [21] and [23, Example 5]. Its mask is given by

$$
\mathrm{a}=\frac{1}{16}\left(\begin{array}{ccccccc}
0 & 0 & 0 & 0 & -1 & -1 & 0 \\
0 & 0 & -1 & 0 & 2 & 0 & -1 \\
0 & -1 & 2 & 8 & 8 & 2 & -1 \\
0 & 0 & 8 & 16 & 8 & 0 & 0 \\
-1 & 2 & 8 & 8 & 2 & -1 & 0 \\
-1 & 0 & 2 & 0 & -1 & 0 & 0 \\
0 & -1 & -1 & 0 & 0 & 0 & 0
\end{array}\right)
$$


It is an interpolating scheme, and reproduces polynomials of degree $k-1=3$. The representation of the mask symbol in terms of three-directional box spline symbols from the list $\mathrm{I}_{4}$ is given by

$$
\begin{array}{r}
z_{1}^{3} z_{2}^{3} \mathrm{a}\left(z_{1}, z_{2}\right) \\
=4\left\{26 \frac{7+6 z_{1} z_{2}}{13} B_{3,3,1}^{\#}\left(z_{1}, z_{2}\right)-2 z_{2} B_{3,1,3}^{\#}\left(z_{1}, z_{2}\right)-2 z_{1} B_{1,3,3}^{\#}\left(z_{1}, z_{2}\right)\right. \\
=4\left\{7 z_{1} z_{2} B_{2,2,2}^{\#}\left(z_{1}, z_{2}\right)\right. \\
\left.\quad-21 \frac{1+z_{1}+z_{2}}{3} B_{2,2,2}^{\#}\left(z_{1}, z_{2}\right)\right\} \\
\left.\quad-2 z_{1,3,3}^{\#}\left(z_{1}, z_{2}\right)-2 z_{2} B_{3,1,3}^{\#}\left(z_{1}, z_{2}\right)-2 z_{1} z_{2} B_{3,3,1}^{\#}\left(z_{1}, z_{2}\right)\right\} .
\end{array}
$$

We see that the generators are all multiples of $B_{1,1,1}^{\#}\left(z_{1}, z_{2}\right)$. This tells us that the symbol can be factorized as

$$
\mathrm{a}\left(z_{1}, z_{2}\right)=B_{1,1,1}^{\#}\left(z_{1}, z_{2}\right) \mathrm{b}\left(z_{1}, z_{2}\right),
$$

a fact noticed in 21]. We would like to emphasize the following properties of the butterfly scheme. Firstly, a simple computation yields that the symbol b(z) does not define a convergent subdivision scheme, although each of the summands in $\mathrm{b}(\boldsymbol{z})$ by itself does correspond to a convergent scheme. Secondly, butterfly is an interpolatory subdivision scheme, but none of the summands in the affine combination above possess this property.

\subsection{A convergent scheme}

The symbols presented in the above examples all possess a property that is very important for their regularity analysis: they are multiples of one specific box spline symbol of type $B_{\alpha, \beta, \gamma}^{\#}$. The regularity analysis of such schemes is given in [19, Section 4.3]. The type of factorization used there, however, is a very special situation which does not generally hold for convergent schemes.

A very simple example that comes to mind is the symbol given by

$$
\begin{aligned}
\mathrm{a}\left(z_{1}, z_{2}\right) & =4\left\{\frac{1}{2} B_{1,1,0}^{\#}\left(z_{1}, z_{2}\right)+\frac{1}{2} B_{0,1,2}^{\#}\left(z_{1}, z_{2}\right)\right\}=4 \frac{1+z_{2}}{2} \mathrm{c}\left(z_{1}, z_{2}\right) \\
\text { with } \quad \mathrm{c}\left(z_{1}, z_{2}\right) & =\frac{1}{2} \frac{1+z_{1}}{2}+\frac{1}{2}\left(\frac{1+z_{1} z_{2}}{2}\right)^{2} .
\end{aligned}
$$

By Theorem 3.2, the symbol a $(\boldsymbol{z})$ is in $\mathcal{I}$, but none of the generators from the list $\mathrm{I}_{1}$ divides the symbol.

In order to check the convergence of this scheme, we study the properties of the so-called difference scheme $\mathcal{S}_{\mathrm{B}}$, see [4, 31], with the matrix mask symbol $\mathrm{B}(\boldsymbol{z})$ satisfying

$$
\mathrm{a}(\boldsymbol{z})\left(\begin{array}{c}
1-z_{1} \\
1-z_{2}
\end{array}\right)^{T}=\left(\begin{array}{c}
1-z_{1}^{2} \\
1-z_{2}^{2}
\end{array}\right)^{T} \mathrm{~B}(\boldsymbol{z}) \text {. }
$$


One possible such $\mathrm{B}(\boldsymbol{z})$ is given by

$$
\begin{aligned}
\mathrm{B}\left(z_{1}, z_{2}\right) & =\left(\begin{array}{ll}
\mathrm{b}_{11}\left(z_{1}, z_{2}\right) & \mathrm{b}_{12}\left(z_{1}, z_{2}\right) \\
\mathrm{b}_{21}\left(z_{1}, z_{2}\right) & \mathrm{b}_{22}\left(z_{1}, z_{2}\right)
\end{array}\right) \\
\text { with } \quad \mathrm{b}_{11}\left(z_{1}, z_{2}\right) & =\frac{1}{4}\left(z_{1} z_{2}^{3}-z_{2}^{3}+z_{1} z_{2}^{2}+z_{2}^{2}+4 z_{2}+2\right), \\
\mathrm{b}_{12}\left(z_{1}, z_{2}\right) & =0 \\
\mathrm{~b}_{21}\left(z_{1}, z_{2}\right) & =\frac{1}{4}\left(z_{1} z_{2}-z_{1}-z_{2}+1\right) \\
\text { and } \quad \mathrm{b}_{22}\left(z_{1}, z_{2}\right) & =\frac{1}{4}\left(z_{1}^{2} z_{2}^{2}+2 z_{1} z_{2}+2 z_{1}+3\right)
\end{aligned}
$$

To check the convergence, we have to verify that the vector subdivision scheme $\mathcal{S}_{\mathrm{B}}$ converges to zero, see [6, 17]. The symbolic calculations yield $\left\|\mathcal{S}_{\mathrm{B}}^{5}\right\|_{\infty}<1$ and, thus, that $\mathcal{S}_{\mathrm{a}}$ is $C$-convergent.

It is worth noting that in this example the two building blocks, with symbols $4 B_{1,1,0}^{\#}\left(z_{1}, z_{2}\right)$ and $4 B_{0,1,2}^{\#}\left(z_{1}, z_{2}\right)$, are not the symbols of $C$-convergent subdivision schemes, while the combination yields $C$-convergence.

We also refer to the constructions in [10], where the convex combination of a four-directional, zero order box spline and a $C^{1}$-quadratic box spline are used to obtain the so-called GP pseudo-quadratic box spline. This example shows enhancement with respect to linear independence of the translates, at the expense of reduced joint smoothness.

\section{References}

[1] C. de Boor and K. Höllig, B-Splines from parallelepipeds, J. Anal. Math. 42 (1983), 99-115.

[2] C. de Boor and K. Höllig, Bivariate box splines and smooth pp functions on a three direction mesh, J. Comput. Appl. Math. 9 (1983), 13-28.

[3] C. de Boor, K. Höllig, and S. D. Riemenschneider, Box Splines, Appl. Math. Sci., vol. 98, Springer-Verlag, New York, 1993.

[4] A. S. Cavaretta, W. Dahmen, and C. A. Micchelli, Stationary Subdivision, Mem. Amer. Math. Soc. 453 (1991).

[5] M. Charina, C. Conti, K. Jetter, and G. Zimmermann, Scalar multivariate subdivision schemes and box splines, Ergebnisberichte Angewandte Mathematik No. 397, Fakultät für Mathematik, Technische Universität Dortmund, Germany, 2009.

[6] M. Charina, C. Conti and T. Sauer, Regularity of multivariate vector subdivision schemes, Numer. Algorithms 39 (2005), 97-113.

[7] O. Christensen, An Introduction to Frames and Riesz Bases, BirkhäuserVerlag, Basel, 2003. 
[8] C. K. Chui, Multivariate Splines, CBMS-NSF Regional Conference Series in Applied Mathematics, vol. 54, SIAM, Philadelphia, 1988.

[9] C. Conti, Stationary and non stationary affine combination of subdivision masks, Math. Comput. Simulation, doi:10.1016/j.matcom.2009.11.004

[10] C. Conti, L. Gori, F. Pitolli, and P. Sablonnière, Approximation by GPbox-splines on a four-direction mesh, J. Comput. Appl. Math. 221 (2008), 310-329.

[11] C. Conti and L. Romani, Affine combination of B-spline subdivision masks and its non-stationary counterparts, submitted (2009).

[12] M. Cotronei and T. Sauer, Full rank filters and polynomial reproduction, Comm. Pure Appl. Math. 6 (2007), 667-687.

[13] I. Daubechies, Ten Lectures on Wavelets, CBMS-NSF Regional Conference Series in Applied Mathematics, vol. 61, SIAM, Philadelphia, 1992.

[14] I. Daubechies, Orthonormal bases of compactly supported wavelets, Comm. Pure Appl. Math. 41 (1988), 909-996.

[15] G. Deslauriers and S. Dubuc, Symmetric iterative interpolation processes, Constr. Approx. 5 (1989), 49-68.

[16] B. Dong and Z. Shen, Pseudo-splines, wavelets and framelets, Appl. Comput. Harmon. Anal. 22 (2007), 78-104.

[17] N. Dyn, Subdivision schemes in computer-aided geometric design, in: W. Light (ed.), Advances in Numerical Analysis. Vol. II: Wavelets, Subdivision Algorithms, and Radial Basis Functions, Clarendon Press, Oxford, 1992, 36-104.

[18] N. Dyn, J. Gregory, and D. Levin, A 4-point interpolatory subdivision scheme for curve design, Comput. Aided Geom. Design 4 (1987), 257-268.

[19] N. Dyn and D. Levin, Subdivision schemes in geometric modelling, Acta Numer. 11 (2002), 73-144.

[20] N. Dyn, K. Hormann, M. A. Sabin, and Z. Shen, Polynomial reproduction by symmetric subdivision schemes, J. Approx. Theory 155 (2008), 28-42.

[21] N. Dyn, D. Levin, and C. A. Micchelli, Using parameters to increase smoothness of curves and surfaces generated by subdivision, Comput. Aided Geom. Design 7 (1990), 129-140.

[22] B. Han, Vector cascade algorithm and refinable function vectors in Sobolev spaces, J. Approx. Theory 124 (2003), 44-88.

[23] B. Han, Classification and construction of bivariate subdivision schemes, in: A. Cohen, J.-L. Merrien, and L. L. Schumaker (eds.), Curve and Surface Fitting, Proc. Saint-Malo 2002, Nashboro Press, Brentwood, 2003, 187-197. 
[24] B. Han and R. Q. Jia, Multivariate refinement equations and convergence of subdivision schemes, SIAM J. Math. Anal. 29 (1998), 1177-1199.

[25] K. Jetter and G. Plonka, A survey on $L_{2}$-approximation orders from shiftinvariant spaces, in: N. Dyn, D. Leviatan, D. Levin, and A. Pinkus (eds.), Multivariate Approximation and Applications, Cambridge University Press, Cambridge, 2001, 73-111.

[26] R.-Q. Jia, Q. Jiang, and S. L. Lee, Convergence of cascade algorithm in Sobolev spaces and integrals of wavelets, Numer. Math. 91 (2002), 453-473.

[27] M.-J. Lai and L. L. Schumaker, Spline Functions on Triangulations, Encyclopedia Math. Appl., vol. 110, Cambridge University Press, Cambridge, 2007.

[28] H. M. Möller and T. Sauer, Multivariate refinable functions of high approximation order via quotient ideals of Laurent polynomials, Adv. Comput. Math. 20 (2004), 205-228.

[29] G. Plonka, Approximation order provided by refinable function vectors, Constr. Approx. 13 (1997), 221-224.

[30] T. Sauer, Polynomial interpolation, ideals and approximation order of multivariate refinable functions, Proc. Amer. Math. Soc. 130 (2002), 33353347.

[31] T. Sauer, Stationary vector subdivision: quotient ideals, differences and approximation power, Rev. R. Acad. Cien. Serie A. Mat. 96 (2002), 257277 .

[32] G. Strang and T. Nguyen, Wavelets and Filter Banks, Wellesley-Cambridge Press, Wellesley, 1996. 